\title{
Updated Absolute Gravity Rate of Change Associated With Glacial Isostatic Adjustment in Southeast Alaska and Its Utilization for Rheological Parameter Estimation
}

\section{Kazuhiro Naganawa ( $\nabla$ naganawa.kazuhiro.66x@st.kyoto-u.ac.jp )}

Kyoto University Graduate School of Science Faculty of Science: Kyoto Daigaku Rigaku Kenkyuka Rigakubu https://orcid.org/0000-0002-5062-8045

\section{Takahito Kazama}

Kyoto University Graduate School of Science Faculty of Science: Kyoto Daigaku Rigaku Kenkyuka Rigakubu

\section{Yoichi Fukuda}

National Institute of Polar Research Division for Research and Education: Kokuritsu Kyokuchi Kenkyujo Kenkyu Kyoikukei

\section{Satoshi Miura}

Tohoku University Graduate School of Science Faculty of Science: Tohoku Daigaku Daigakuin Rigaku Kenkyuka Rigakubu

\section{Hideki Hayakawa}

The University of Tokyo: Tokyo Daigaku

\section{Yusaku Ohta}

Tohoku University Graduate School of Science Faculty of Science: Tohoku Daigaku Daigakuin Rigaku Kenkyuka Rigakubu Jeffrey Freymueller Michigan State University

\section{Research Article}

Keywords: absolute gravity change, Southeast Alaska, rheological parameter, mantle viscosity, lithospheric thickness, present-day ice melting, Little Ice Age, vertical gravity gradient

Posted Date: December 20th, 2021

DOI: https://doi.org/10.21203/rs.3.rs-1158163/v1

License: (c) (1) This work is licensed under a Creative Commons Attribution 4.0 International License. 



\section{Updated absolute gravity rate of change associated with glacial}

2 isostatic adjustment in Southeast Alaska and its utilization for ${ }_{3}$ rheological parameter estimation

${ }_{4}$ Kazuhiro Naganawa*, Graduate School of Science, Kyoto University, Sakyo-ku, Kyoto 6068502, Japan, naganawa.kazuhiro.66x@st.kyoto-u.ac.jp

Takahito Kazama, Graduate School of Science, Kyoto University, Sakyo-ku, Kyoto 606-8502, Japan, takujin@kugi.kyoto-u.ac.jp

Yoichi Fukuda, Graduate School of Science, Kyoto University, Sakyo-ku, Kyoto 606-8502, Japan; Now at National Institute of Polar Research, Tachikawa, Tokyo 190-8518, Japan, fukuda.yoichi@nipr.ac.jp

Satoshi Miura, Research Center for Prediction of Earthquakes and Volcanic Eruptions, Graduate School of Science, Tohoku University, Aoba-ku, Sendai, Miyagi 980-8572, Japan, miura3104@tohoku.ac.jp

Hideaki Hayakawa, Institute for Cosmic Ray Research, the University of Tokyo, Kashiwa, Chiba 277-8582, Japan, haya@icrr.u-tokyo.ac.jp

Yusaku Ohta, Research Center for Prediction of Earthquakes and Volcanic Eruptions, Graduate School of Science, Tohoku University, Aoba-ku, Sendai, Miyagi 980-8572, Japan, yusaku.ohta.d2@tohoku.ac.jp Jeffrey T. Freymueller, Geophysical Institute, University of Alaska Fairbanks,2156 Koyukuk Drive, Fairbanks, AK 99775, USA; Now at Department of Earth and Environmental Sciences, Michigan State University, East Lansing, MI 48824, USA, freymuel@msu.edu 


\section{Abstract}

7 In Southeast Alaska (SE-AK), rapid ground uplift of up to $3 \mathrm{~cm} / \mathrm{yr}$ has been observed associated with

8 post-Little Ice Age glacial isostatic adjustment (GIA). Geodetic techniques such as global navigation

9 satellite system (GNSS) and absolute gravimetry have been applied to monitor GIA since the last

10

11 1990s. Rheological parameters for SE-AK were determined from dense GNSS array data in earlier studies. However, the absolute gravity rate of change observed in SE-AK was inconsistent with the ground uplift rate, mainly because few gravity measurements from 2006 to 2008 resulted in imprecise gravity variation rates. Therefore, we collected absolute gravity data at six gravity points in SE-AK every June in 2012, 2013, and 2015, and updated the gravity variation rate by reprocessing the absolute gravity data collected from 2006 to 2015 . We found that the updated gravity variation rate at the six gravity points ranged from -2.05 to $-4.40 \mu \mathrm{Gal} / \mathrm{yr}$, and its standard deviation was smaller than that reported in the earlier study by up to $88 \%$. We also estimated the rheological parameters to explain the updated gravity variation rate, and their optimal values were determined to be $55 \mathrm{~km}$ and $1.2 \times 10^{19} \mathrm{~Pa}$ s for lithospheric thickness and upper mantle viscosity, respectively. These optimal values are consistent with those independently obtained from GNSS observations, and this fact indicates that absolute gravimetry can be one of the most effective methods in determining sub-surface structural parameters associated with GIA accurately. Moreover, we utilized the gravity variation rates for estimating the ratio of gravity variation to vertical ground deformation at the six gravity points in SE-AK. The viscous ratio values were obtained as -0.168 and $-0.171 \mu \mathrm{Gal} / \mathrm{mm}$ from the observed data and the calculated result, respectively. These ratios are greater (in absolute) than those for other GIA regions $(-0.15$ to $-0.16 \mu \mathrm{Gal} / \mathrm{mm}$ in Antarctica and Fennoscandia) because glaciers in SE-AK have melted more recently than in other regions.

\section{Keywords}

absolute gravity change, Southeast Alaska, rheological parameter, mantle viscosity, lithospheric thickness, present-day ice melting, Little Ice Age, vertical gravity gradient 


\section{Introduction}

In Southeast Alaska (SE-AK), significant masses of glaciers have melted since the end of the Little Ice Age (LIA) in the 19-20th centuries, and the glacier melting is still ongoing. Rapid ground uplift of up to $3 \mathrm{~cm} / \mathrm{yr}$ has been observed at the global navigation satellite system (GNSS) stations in this region (Fig. 1; Larsen et al 2004), which is mainly caused by Glacial Isostatic Adjustment (GIA; i.e., the elastic/viscoelastic response of solid earth to present/past glacier melting). Since 1998, GNSS stations have been surveyed in SE-AK to determine the spatiotemporal variations in the uplift rate more precisely (Larsen et al 2005). The observed uplift rates were quantitatively reproduced using GIA models, which are composed of spatiotemporal ice load distributions and medium parameters indicating a thin lithosphere $(\sim 60 \mathrm{~km})$ and low-viscosity asthenosphere $\left(\sim 10^{19} \mathrm{~Pa} \mathrm{~s}\right)$ (Larsen et al 2004, 2005; Elliott et al 2010; Sato et al 2011; Hu and Freymueller 2019).

Sun et al (2010) also measured absolute gravity (AG) values on the ground in SE-AK every June from 2006 to 2008 as part of the Internatinal geodetic project in South-Eastern Alaska (ISEA) (FY2005-2008) to reveal the spatiotemporal mass variations associated with GIA and present-day ice melting (PDIM) directly. They determined the AG values at five gravity points (red circles in Fig. 1) within 1- $\mu$ Gal precision, and found a linear gravity decrease of up to $-5.6 \mu \mathrm{Gal} / \mathrm{yr}$. Sato et al (2012) additionally determined the AG variation rate at Blanchard Road Maintenance Compound (BRMC) (red square in Fig. 1) to be $-2.9 \mu \mathrm{Gal} / \mathrm{yr}$ using $\mathrm{AG}$ data collected in June 2007 and 2008, and reproduced the AG variation rates at six gravity points (red circles and squares in Fig. 1) using their GIA model (Sato et al 2011).

However, the following three problems still exist for all the earlier studies on gravity variations in SE-AK (Sun et al 2010; Sato et al 2012). First, a three-year duration is too short to obtain precise AG variation rates. In the earlier studies, 1- $\sigma$ error values of the AG variation rates at five gravity points were estimated to be $0.4-2.9 \mu \mathrm{Gal} / \mathrm{yr}$, which corresponded to $9-57 \%$ of the absolute values of the AG variation rates (Sun et al 2010). If AG values are newly measured at the same gravity points after an interval of several years, more precise variation rates can be obtained. Second, Sato et al $(2011,2012)$ decreased the resolution of PDIM models (Larsen et al 2005, 2007) on purpose to reduce the computational time. Since gravity change is sensitive to the spatiotemporal variation in ice melting, high-resolution PDIM models should be prepared to calculate gravity variations accurately. Third, none of the earlier studies have created GIA models from the AG variation rate itself; for example, Sato et al (2012) simply reproduced the observed 
AG variation rate using their GIA model, which was based on the GNSS data (Sato et al 2011). Although less AG data are available relative to the GNSS data, a new GIA model can be developed from the AG data given a longer AG time series, and it should be compared with earlier GNSS-based models (e.g., Sato et al 2011; Hu and Freymueller 2019).

Thus, we constructed a new GIA model using high-resolution PDIM models and an updated data set of AG observations from 2006 to 2015. New AG measurements were collected at six gravity points in SE-AK every June in 2012, 2013, and 2015 as part of the ISEA2 project (FY2011-2015), and all of the AG data from 2006 to 2015 were reprocessed using a consistent procedure. AG variation rates were obtained from the AG data set from 2006 to 2015 after correcting for hydrological and coseismic gravity changes. The obtained AG variation rates were quantitatively explained by numerical calculations of gravity changes derived from GIA and PDIM. High-resolution PDIM models were then utilized, and optimal medium parameters such as lithospheric thickness and asthenospheric viscosity were determined. The coherency of the medium parameters was finally discussed by comparing the observed uplift rate with the one calculated from our GIA model.

\section{Absolute gravity data}

We conducted repeat AG measurements at six gravity points (red symbols in Fig. 1) in June 2012, 2013, and 2015 using an FG5 absolute gravimeter (serial number: 111) (Micro-g LaCoste 2006). Table 1 shows the coordinates and vertical gravity gradient of the AG points, along with coordinates of their nearest continuous GNSS stations. The FG5 gravimeter was set up at most of the AG points according to the Appendix in Sun et al (2010). However, at Mendenhall Glacier Visitor Center (MGVC), we needed to move the AG point by about $5 \mathrm{~m}$ in June 2012 because an exhibit was installed on the old AG point by the visitor center (see Appendix). The gravity difference between the old and new gravity points for MGVC was estimated with the least-square method, as discussed in the next section. The AG data were collected for approximately two days at each point to obtain approximately 100 sets of gravity values. One set was composed of 100 drops of the gravity measurement, and the set and drop intervals were chosen to be $30 \mathrm{~min}$ and $10 \mathrm{~s}$, respectively.

The AG data were then processed using the g9 software (Micro-g LaCoste 2012) with basic gravity corrections applied to each drop gravity data as follows: the effect of air pressure change was corrected using simultaneously obtained pressure data with an admittance factor of $-0.30 \mu \mathrm{Gal} / \mathrm{hPa}$ and nominal 
air pressure values as shown in Table 1; the solid earth tide was corrected using the ETGTAB software (Timmen and Wenzel 1995) with the delta factor for permanent tide of 1.0; the polar motion effect was corrected using polar position values provided by International Earth Rotation and Reference Systems Service (IERS) Bulletin A and a delta factor of 1.164; the AG value at a hight of $100 \mathrm{~cm}$ from the benchmark of each AG point was estimated through correction of the instrumental height difference using the gravity gradient values as shown in Table 1. The ocean-tide loading effect was also corrected with the regional ocean-tide model developed by Inazu et al (2009), but we found that diurnal and semidiurnal gravity changes persisted in the time series of set gravity at seashore AG points (Fig. 2a) due to the under-correction of the ocean-tide effect. Although the ocean-tide model should be improved in future studies, we applied a regression of the diurnal and semidiurnal sine curves to the set gravity data to estimate the average gravity value at each AG point for each year (Fig. 2).

Moreover, we reprocessed past AG data collected from 2006 to 2008 (Sun et al 2010; Sato et al 2012) using the same procedures as above, and obtained a new dataset of the AG values at six gravity points in SE-AK from 2006 to 2015 (Table 2). The AG values are observed to decrease at all gravity points, mainly due to the past/present ice melting, which was modeled and will be discussed later in the article. However, at the AG point located in Russell Island (RUSG), the AG value in 2012 largely deviated from the decreasing trend because the AG data still contained the effect of ground vibration due to the failure of a long-period spring in the FG5-111 gravimeter.

\section{Absolute gravity rate of change}

\section{Effect of hydrological gravity disturbances}

Since temporal AG variations can be caused by annual hydrological storage, its effect should be corrected from the observed AG data to quantify AG variation associated with GIA and PDIM. Although the effect is expected to be minimized due to the AG data being measured every June in the case of SEAK (see the previous section), multi-year variations in land-water storage may be detected by the AG measurements. The SNOw TELemetry (SNOTEL) data at Long Lake (green triangle in Fig. 1) showed less snow accumulation in 2006 and 2015, compared with those in the other years when the AG data were collected (Fig. 3a). This implies that the observed AG values should decrease in 2006 and 2015 because less snow mass leads to smaller attraction force and greater unloading uplift.

Thus, we estimated gravity variations due to hydrological loading (Farrell 1972) and mass attraction $\left(g_{e}\right.$ 
and $g_{a}$, respectively) at each gravity point:

$$
\begin{aligned}
& g_{e}\left(\theta_{p}, \lambda_{p}, t\right)=\rho_{w} \int H_{w}(\theta, \lambda, t) G_{g e}(\varphi) d S \\
& g_{a}\left(\theta_{p}, \lambda_{p}, t\right)=\rho_{w} \int H_{w}(\theta, \lambda, t) G_{a}\left(\theta, \lambda ; \theta_{p}, \lambda_{p}\right) d S,
\end{aligned}
$$

where $\left(\theta_{p}, \lambda_{p}\right)$ and $(\theta, \lambda)$ indicate the coordinates (latitude and longitude) of the gravity point and a loading point, respectively. $\rho_{w}, H_{w}(\theta, \lambda, t), G_{g e}(\varphi), \varphi=\varphi\left(\theta, \lambda ; \theta_{p}, \lambda_{p}\right)$, and $G_{a}\left(\theta, \lambda ; \theta_{p}, \lambda_{p}\right)$ also indicate the water density, the spatiotemporal distributions in water thickness, the elastic Green's function for gravity change due to point loading, the angular distance between $\left(\theta_{p}, \lambda_{p}\right)$ and $(\theta, \lambda)$, and the Green's function for gravity change due to mass attraction, respectively. $G_{g e}(\varphi)$ was first prepared by linearly interpolating the original Green's function of the 1066A Earth model (Gilbert and Dziewonski 1975; Matsumoto et al 2001) to obtain the loading response for each angular distance. $G_{a}\left(\theta, \lambda ; \theta_{p}, \lambda_{p}\right)$ was also prepared by considering the Earth's curvature and topography:

$$
G_{a}\left(\theta, \lambda ; \theta_{p}, \lambda_{p}\right)=\frac{G}{r^{2}} \cdot \frac{r^{2}+h_{p}^{2}-\{h(\theta, \lambda)\}^{2}}{2 r h_{p}},
$$

where $G, r=r\left(\theta, \lambda, h ; \theta_{p}, \lambda_{p}, h_{p}\right), h(\theta, \lambda)$, and $h_{p}$ indicate gravitational constant, the direct distance between the gravity and loading points, and the geocentric distances of the gravity and loading points, respectively. Here, we assumed that each hydrological mass was located on the corresponding cell of the ground, as expressed by the ETOPO1 digital elevation model (Amante and Eakins 2009). These Green's functions were then multiplied by $\rho_{w} H(\theta, \lambda, t)$, i.e., the water density value multiplied by the spatiotemporal distributions of soil moisture, snow water equivalent and canopy in the Global Land Data Assimilation System version 2.1 (GLDAS-2.1) dataset (Rodell et al 2004). The gravity responses were finally estimated by spatially integrating the product of Green's functions and water distribution (Eq. 12); cell sizes of the water distributions were defined as $0.0025^{2}, 0.025^{2}$, and $0.25^{2} \operatorname{deg}^{2}$ for $0.004 \leq \varphi<1.0$, $1.0 \leq \varphi<2.0$, and $2.0 \leq \varphi \mathrm{deg}$, respectively, and the water distributions in $\varphi<0.004$ were excluded from the integral calculations to avoid singularity at $\varphi \sim 0$ deg.

Figure 3b shows the estimated gravity variations due to soil moisture, snow, and canopy (top, middle, and bottom of the panel, respectively) at the EGAN gravity point (Fig. 1). Red, green, and blue lines for each hydrological component indicate the loading effect $\left(g_{e}\right)$, the attraction effect $\left(g_{a}\right)$, and the sum of the two effects $\left(g_{e}+g_{a}\right)$, respectively. Most of the gravity time series change annually, reaching a maximum in winter, mainly because of significant snow mass accumulation around SE-AK in winter (Fig. 3a). In contrast, the gravity variation due to snow mass attraction $\left(g_{a}\right.$, green line in the middle of Fig. $\left.3 \mathrm{~b}\right)$ 
reaches a minimum in winter because accumulated snow in high mountains causes on upward attraction force (i.e., gravity decrease) to a gravity point located at the foot of the mountains.

The blue lines in Figure 3c show the sum of $g_{e}+g_{a}$ for the three hydrological components (blue lines in Fig. 3b) at six gravity points (Fig. 1). The gravity time series at most of the gravity points reach a maximum in winter mainly due to snow loading (see Fig. 3b), although the gravity value is minimum in winter at MGVC due to the strong upward attraction force caused by snow mass accumulated on alpine glaciers near MGVC. Blue circles in Figure 3c also indicate the hydrological gravity changes expected during the periods when $\mathrm{AG}$ values were measured in SE-AK; their variation range is estimated to be approximately $2.5 \mu \mathrm{Gal}$ at $\mathrm{MGVC}$ and less than $1 \mu \mathrm{Gal}$ at the other gravity points.

\section{Effect of coseismic gravity changes}

Coseismic gravity changes should also be corrected with the observed AG data to quantify the AG variation associated with GIA and PDIM. During the observation period from 2006 to 2015 , two large earthquakes occurred near SE-AK as follows: [1] The Mw 7.8 Haida Gwaii earthquake occurred on 28 October 2012 because of slip on a low-angle thrust fault off the west coast of Moresby Island, Haida Gwaii (Nykolaishen et al 2015). Its epicenter was located approximately $640 \mathrm{~km}$ from the EGAN AG point (Fig. 4a). [2] The Mw 7.5 Craig Earthquake occurred on 5 January 2013 because of the strike slip along the Queen Charlotte Fault off the coast of SE-AK (Lay et al 2013). Its epicenter was located approximately $290 \mathrm{~km}$ from the EGAN AG point (Fig. 4b).

Figure $4 \mathrm{c}-\mathrm{d}$ shows the coseismic gravity changes due to these two earthquakes, calculated on the basis of the dislocation theory of Okubo (1992) and slip distribution models by Lay et al (2013). A small gravity increase of up to $0.15 \mu \mathrm{Gal}$ was expected at the AG points during the 2012 Haida Gwaii earthquake (Fig. 4c) mainly because of the small ground subsidence at the AG points caused by the coseismic slip. In contrast, a significant gravity decrease of up to $1.2 \mu \mathrm{Gal}$ was expected during the 2013 Craig Earthquake (Fig. 4d) because the coseismic slip in the vicinity of SE-AK led to a ground uplift of up to $4 \mathrm{~mm}$ at the AG points.

We disregarded temporal gravity changes due to postseismic deformations. In fact, post-seismic vertical displacement of up to $6 \mathrm{~mm}$ was observed near the epicenter of the Haida Gwaii earthquake (Nykolaishen et al 2015), although no significant post-seismic displacement was detected around our AG points in the SE-AK area, according to the GNSS time series obtained by Nevada Geodetic Laboratory (Blewitt et al 2018). 


\section{Updated gravity variation rate}

Red circles in Figure 5 indicate the AG values at the six gravity points after the effects of hydrology and the two earthquakes were corrected in the original AG data (gray circles). The correction value is typically less than $1 \mu \mathrm{Gal}$, but it is as large as $1.80 \mu \mathrm{Gal}$ at EGAN in 2013 mainly because of the coseismic gravity decrease owing to the 2013 Craig earthquake (Fig. 4d).

We calculated a new gravity variation rate at each AG point from the corrected AG values from 2006 to 2015 (red circles in Fig. 5) using the weighted least-squares method. The weight of each AG data was defined by the reciprocal of the squared sum of the standard deviation (SD) and uncertainty values (see Table 2). Additionally, we calculated a gravity step at MGVC between 2008 and 2012 in the least-squares calculation because we had to relocate the AG point by about $5 \mathrm{~m}$ in June 2012 due to an exhibit installed on the old AG point by the visitor center (see Appendix). We also excluded the AG value obtained at RUSG in 2012 because it contained a large error associated with the failure of the FG5-111 gravimeter (see the section of "Absolute gravity data").

The red lines in Figure 5 indicate the updated AG variation rates at the six gravity points. Our variation rates were estimated with smaller error values (see the right side of Figure 5) than those obtained in earlier studies (Sun et al 2010; Sato et al 2012) at most gravity points because new AG data from 2012 to 2015 were added to estimate the AG variation rate. However, the error of the variation rate at MGVC $( \pm 1.06 \mu \mathrm{Gal} / \mathrm{yr})$ was about twice as great as that obtained by Sun et al (2010) because the AG values from 2012 to 2015 deviated from the linear gravity trend. The deviation of the AG data also caused the poor estimation of the gravity step associated with the relocation of the MGVC gravity point $(+3.53 \pm$ $7.01 \mu \mathrm{Gal})$.

At most of the AG points, the updated gravity variation rates are smaller by up to $2.8 \mu \mathrm{Gal} / \mathrm{yr}$, than that reported by the earlier studies (Sun et al 2010; Sato et al 2012). The gravity variation rate is greatest at RUSG in all of the AG points; this result is consistent with the spatial pattern of uplift associated with the GIA and PDIM (Fig. 1). The difference of the gravity variation rates between EGAN and MGVC reaches approximately $0.8 \mu \mathrm{Gal} / \mathrm{yr}$ despite the close distance (approximately $6.6 \mathrm{~km}$; see Fig. 1), probably because the glacier mass loss on the top of the mountain glaciers can decrease the upward attraction force (i.e., increase the gravity value) at MGVC, which is located at the foot of Mendenhall Glacier (see Appendix). 


\section{Numerical calculation of gravity variation rate}

The observed gravity variation rates (Fig. 5) were then compared with those calculated using the GIA/PDIM models. In the case of SE-AK, the GIA source can be separated into four components: global past ice melting (GPIM), regional past ice melting (RPIM), local past ice melting (LPIM) and present-day ice melting (PDIM). We numerically calculated the gravity variation rates due to four GIA components, along with the time variation in attraction force due to PDIM.

\section{The effect of global past ice melting (GPIM)}

Rate of temporal gravity variation due to past ice melting (denoted by $\dot{g}_{v}$ ) can be calculated using the load deformation theory for the viscoelastic medium (Peltier 1974):

$$
\dot{g}_{v}\left(\theta_{p}, \lambda_{p}, t_{0}\right)=\rho_{i} \iint \Delta H_{i}(\theta, \lambda, s) \dot{G}_{g v}\left(\varphi, t_{0}-s\right) d S d s
$$

where $t_{0}, \rho_{i}$, and $\Delta H_{i}(\theta, \lambda, s)$ indicate the present time, the typical glacier density $\left(=850 \mathrm{~kg} / \mathrm{m}^{3} ; \mathrm{Huss}\right.$ 2013; Hu and Freymueller 2019), and the spatiotemporal distribution in glacier melting at a time $s$, respectively. $\dot{G}_{g v}\left(\varphi, t_{0}-s\right)$ also indicate the time derivative of the viscoelastic Green's function for gravity variation at a time $t_{0}$ caused by the point loading on the Maxwell Earth at a time $s$ :

$$
\begin{aligned}
\dot{G}_{g v}\left(\varphi, t_{0}-s\right) & =\frac{g_{0}}{m_{e}} \sum_{n=0}^{n_{\max }} \dot{\delta}_{n}\left(t_{0}-s\right) P_{n}(\cos \varphi) \\
& =\frac{g_{0}}{m_{e}} \sum_{n=0}^{n_{\max }}\left\{-2 \dot{h}_{n}\left(t_{0}-s\right)+(n+1) \dot{k}_{n}\left(t_{0}-s\right)\right\} P_{n}(\cos \varphi)
\end{aligned}
$$

where $g_{0}$ and $m_{e}$ indicate the typical absolute gravity values $\left(=9.8065 \mathrm{~m} / \mathrm{s}^{2}\right)$ and Earth's mass (= $\left.5.97 \times 10^{24} \mathrm{~kg}\right)$, respectively. $\dot{h}_{n}, \dot{k}_{n}$ and $P_{n}$ also indicate the time derivatives of loading Love numbers and the Legendre polynomial for the $n$-th degree, respectively. $\dot{\delta}_{n}$ and $\dot{h}_{n}$ are proportional to the rates of gravity variation and vertical deformation to unit loading, respectively (see Eqs. 14-15). The loading Love numbers $\left(\dot{h}_{n}\right.$ and $\left.\dot{k}_{n}\right)$ were calculated using the ALMA software (Spada 2008), with the assumption of a spherically symmetric, incompressible, and Maxwell viscoelastic Earth. The summation of Equation (5) was performed up to the degree of $n_{\max }=180$ because the loading response to past ice melting converges to its asymptotic value at a degree of $n \sim 130$ (e.g., Fig. 4 in Sato et al 2011).

We estimated the present-day gravity variation due to the GPIM for each gravity point $p$ (denoted by $\left.\dot{g}_{v}^{G P I M}\left(\theta_{p}, \lambda_{p}, t_{0}\right)\right)$, using the global historical deglaciation model ICE-6G_C (Peltier et al 2015) for $\Delta H_{i}$ in Equation (4). We also calculated the loading Love numbers ( $\dot{h}_{n}$ and $\dot{k}_{n}$ in Equation 5$)$ by applying the viscosity value in the VM5a model (Peltier et al 2015), and the values of density and shear modulus in the 
Preliminary Reference Earth Model (PREM) model (Dziewonski and Anderson 1981) using the ALMA software (Spada 2008). The loading Love numbers and resultant Green's function $\left(\dot{G}_{g v}\right.$ in Equations $\left.4-5\right)$ were calculated at the time step of $0.5 \mathrm{kyr}$, which is consistent with that for the ICE-6G_C deglaciation model.

Figure $6 \mathrm{a}-\mathrm{c}$ indicate the behavior of $-\dot{\delta}_{n}(\tau)$ and $\dot{h}_{n}(\tau)$ to the spherical harmonic degree $(n)$ for the VM5a model, where $\tau=t_{0}-s$ (see Eqs. $\left.4-5\right)$. Both $-\dot{\delta}_{n}(\tau)$ and $\dot{h}_{n}(\tau)$ reach their maxima at $n=30--50$, and the ratio of $-\dot{\delta}_{n}(\tau) / \dot{h}_{n}(\tau)$ is constant in time, despite the temporal decrease in $-\dot{\delta}_{n}(\tau)$ and $\dot{h}_{n}(\tau)$. Figure $6 \mathrm{~d}-\mathrm{f}$ also indicate the variations of $-\dot{\delta}(\varphi, \tau)$ and $\dot{h}(\varphi, \tau)$ to the angular distance from a loading point, $\varphi$ :

$$
\begin{aligned}
\dot{\delta}(\varphi, \tau) & =\sum_{n=0}^{n_{\max }} \dot{\delta}_{n}(\tau) P_{n}(\cos \varphi), \\
\dot{h}(\varphi, \tau) & =\sum_{n=0}^{n_{\max }} \dot{h}_{n}(\tau) P_{n}(\cos \varphi)
\end{aligned}
$$

$-\dot{\delta}(\varphi, \tau)$ and $\dot{h}(\varphi, \tau)$ reaches its maximum at $\varphi=0 \mathrm{deg}$, and they decrease with the increments of $\varphi$ and $\tau$. The ratio of $-\dot{\delta}(\varphi, \tau) / \dot{h}(\varphi, \tau)$ also depends on the time, mainly because the position of $\varphi$ where $\dot{h}(\varphi, \tau)=0$ varies with time. However, $-\dot{\delta}(0, \tau) / \dot{h}(0, \tau)$ is nearly constant $(0.16-0.17 \mu \mathrm{Gal} / \mathrm{mm})$ at any time, and its constant value is close to the Bouguer approximation value (e.g., Ekman and Mäkinen 1996; James and Ivins 1998). All of these characteristics about the loading Love numbers agree well with past studies (e.g., Figs. 1-2 in Olsson et al 2015).

\section{The effects of regional/local past ice melting (RPIM and LPIM)}

To accurately calculate gravity variations caused by rapid ice melting since the end of the LIA in SE-AK, we additionally utilize the spatiotemporally high-resolution ice melting models denoted by RPIM and LPIM. The RPIM model is identical to the regional model in Larsen et al (2004, 2005), and is composed of 531 disk loads with a $20 \mathrm{~km}$ diameter in South and Southeast Alaska (see Fig. 2.4 in Larsen 2003). This model was developed by extrapolating the ice melting rate of Arendt et al (2002) to the end of the LIA in 1900. The LPIM model is also identical to the Glacier Bay model in Larsen et al (2004, 2005), and was constructed based on aerophotography and field investigations. This model consists of five disk loads with 26-39 km diameters on the Glacier Bay area (see Fig. 5a in Larsen et al 2005). As the effects of RPIM and LPIM are sensitive to the viscoelastic structure beneath SE-AK, we determine optimum parameters for the structure via the following grid search calculation. We first assume that the asthenosphere is composed of four viscoelastic layers (Table 3), and use the structure model of Sato et al 
(2011) for most of the parameters except for the lithosphere thickness $(d)$ and upper mantle viscosity $(\eta)$.

We also choose a pair of $(d, \eta)$ in the range of $30 \leq d \leq 120[\mathrm{~km}]$ and $0.5 \times 10^{19} \leq \eta \leq 5.0 \times 10^{19}[\mathrm{~Pa} \mathrm{~s}]$, and calculate the loading Love numbers and the consequent Green's function (Eq. 5) using the ALMA software (Spada 2008). In this calculation, PREM (Dziewonski and Anderson 1981) was utilized for the values of the density and shear modulus. The gravity variation rates due to RPIM and LPIM for each gravity point $(p)$ are then estimated according to Equation (4), and are denoted by $\dot{g}_{v}^{R P I M}\left(\theta_{p}, \lambda_{p}, t_{0} ; d, \eta\right)$ and $\dot{g}_{v}^{L P I M}\left(\theta_{p}, \lambda_{p}, t_{0} ; d, \eta\right)$, respectively. During the numerical integral of Equation (4), the disk loads of the RPIM and LPIM models were converted to point loads with a cell size of 0.01 degree. These calculations are repeated for all pairs of $(d, \eta)$ with grid intervals of $\Delta d=10 \mathrm{~km}$ and $\Delta \eta=0.5 \times 10^{19} \mathrm{~Pa}$ $\mathrm{s}$, and the optimum pair of $(d, \eta)$ is finally determined to minimize the $\chi^{2}$ value:

$$
\begin{aligned}
& \chi^{2}(d, \eta)=\sum_{p}\left[\frac{\dot{g}_{o b s}\left(\theta_{p}, \lambda_{p}\right)-\dot{g}_{c a l}\left(\theta_{p}, \lambda_{p}, t_{0} ; d, \eta\right)}{\sigma_{o b s}\left(\theta_{p}, \lambda_{p}\right)}\right]^{2}, \\
& \dot{g}_{c a l}\left(\theta_{p}, \lambda_{p}, t_{0} ; d, \eta\right)= \dot{g}_{v}\left(\theta_{p}, \lambda_{p}, t_{0} ; d, \eta\right)+\dot{g}_{e a}\left(\theta_{p}, \lambda_{p}\right) \\
&= {\left[\dot{g}_{v}^{G P I M}\left(\theta_{p}, \lambda_{p}, t_{0}\right)+\dot{g}_{v}^{\text {RPIM }}\left(\theta_{p}, \lambda_{p}, t_{0} ; d, \eta\right)+\dot{g}_{v}^{L P I M}\left(\theta_{p}, \lambda_{p}, t_{0} ; d, \eta\right)\right] } \\
&+\dot{g}_{e a}^{\text {PDIM }}\left(\theta_{p}, \lambda_{p}\right) .
\end{aligned}
$$

In Equation (8), $\dot{g}_{o b s}\left(\theta_{p}, \lambda_{p}\right)$ and $\sigma_{o b s}\left(\theta_{p}, \lambda_{p}\right)$ indicate the observed gravity variation rate and SD for each gravity point $p$, respectively. In Equation $(9), \dot{g}_{e a}^{P D I M}\left(\theta_{p}, \lambda_{p}\right)$ indicates the gravity variation rate due to PDIM, as described in the next section.

\section{The effects of present-day ice melting (PDIM)}

Gravity variation associated with PDIM can be divided into loading and attraction effects. We calculated the gravity variation rates due to two effects $\left(\dot{g}_{e}\right.$ and $\dot{g}_{a}$, respectively) by referring to Equations (1) and (2):

$$
\begin{aligned}
& \dot{g}_{e}\left(\theta_{p}, \lambda_{p}\right)=\rho_{i} \iint \dot{H}_{i}(\theta, \lambda) G_{g e}(\varphi) d S, \\
& \dot{g}_{a}\left(\theta_{p}, \lambda_{p}\right)=\rho_{i} \iint \dot{H}_{i}(\theta, \lambda) G_{a}\left(\theta, \lambda ; \theta_{p}, \lambda_{p}\right) d S,
\end{aligned}
$$

where $\dot{H}_{i}(\theta, \lambda)$ indicates the spatial distribution of PDIM's rate. In this calculation, we used the same Green's functions $\left(G_{g e}(\varphi)\right.$ and $\left.G_{a}\left(\theta, \lambda ; \theta_{p}, \lambda_{p}\right)\right)$ as for calculating the hydrological gravity variations (see the section titled "Effect of hydrological gravity disturbances").

We utilized the PDIM models of UAF05 (Arendt et al 2002; Larsen et al 2005) and UAF07 (Larsen et al 2007) for $\dot{H}_{i}(\theta, \lambda)$. UAF05 indicates the spatial distribution of ice elevation change from the mid-1950s 
to the mid-1990s, and covers South/Southeast Alaska $\left(-150^{\circ}\right.$ to $-130^{\circ} \mathrm{E}$ and $+55.5^{\circ}$ to $\left.+62.5^{\circ} \mathrm{N}\right)$ with grid intervals of approximately $0.01^{\circ}$ and $0.005^{\circ}$ for longitude and latitude, respectively. UAF07 also indicates the ice thickness change in $\mathrm{SE}-\mathrm{AK}\left(-139.5^{\circ}\right.$ to $-132.0^{\circ} \mathrm{E}$ and $+56.5^{\circ}$ to $\left.+60.0^{\circ} \mathrm{N}\right)$ from 1948 to 2000 , and its grid interval is $0.000556^{\circ}$ for both longitude and latitude. We merged two PDIM models to calculate the PDIM-derived gravity variations (Eqs. 10-11). In the merged PDIM model, UAF07 was used for SE-AK, and UAF05 was additionally used for the rest of South/Southeast Alaska. Therefore, the sum of the PDIM effect can be written as follows:

$$
\begin{aligned}
\dot{g}_{e a}^{P D I M}\left(\theta_{p}, \lambda_{p}\right) & =\dot{g}_{e}^{P D I M}\left(\theta_{p}, \lambda_{p}\right)+\dot{g}_{a}^{\text {PDIM }}\left(\theta_{p}, \lambda_{p}\right) \\
& =\dot{g}_{e}^{U A F 07}\left(\theta_{p}, \lambda_{p}\right)+\dot{g}_{e}^{U A F 05}\left(\theta_{p}, \lambda_{p}\right)+\dot{g}_{a}^{U A F 07}\left(\theta_{p}, \lambda_{p}\right)+\dot{g}_{a}^{U A F 05}\left(\theta_{p}, \lambda_{p}\right) .
\end{aligned}
$$

\section{Results}

\section{Optimal rheological parameters}

Figure 7a shows the distribution of $\chi^{2}$ for the variations in $d$ and $\eta \cdot \chi^{2}$ has a trade-off between $d$ and $\eta$, i.e., for a certain value of $\chi^{2}, d$ decreases as $\eta$ increases. This trade-off can be explained by the positive correlation between asthenospheric viscosity $(\eta)$ and the cube of the asthenospheric thickness (denoted by $D^{3}$ ) for a constant relaxation time value (e.g., Richards and Lenardic 2018). Because $d+D=220 \mathrm{~km}$ in our case (Table 3 ), the synthetic gravity time series and consequent $\chi^{2}$ value remain constant as long as $d$ is negatively correlated to $\eta$. The minimum $\chi^{2}$ value is less than five, and the parameter ranges for $\chi^{2}=5$ are $50 \leq d \leq 60 \mathrm{~km}$ and $1 \times 10^{19} \leq \eta \leq 2 \times 10^{19} \mathrm{~Pa}$ s. However, the grid widths are too coarse $\left(\Delta d=10 \mathrm{~km}\right.$ and $\Delta \eta=0.5 \times 10^{19} \mathrm{~Pa}$ s) to determine an optimum pair of $(d, \eta)$ more precisely.

Figure $7 \mathrm{~b}$ shows the distribution of $\chi^{2}$ obtained from an additional grid search calculation with smaller grid widths $\left(\Delta d=2 \mathrm{~km}\right.$ and $\left.\Delta \eta=0.1 \times 10^{19} \mathrm{~Pa} \mathrm{~s}\right)$. This calculation was applied to the range of $45 \leq d \leq 65 \mathrm{~km}$ and $0.5 \times 10^{19} \leq \eta \leq 2.5 \times 10^{19} \mathrm{~Pa} \mathrm{~s}$ (red squares in Figure 7a). $\chi^{2}$ reaches a minimum value of $\chi_{\text {min }}^{2}=3.39$ at $d=55 \mathrm{~km}$ and $\eta=1.2 \times 10^{19} \mathrm{~Pa} \mathrm{~s}$ (red cross in Fig. 7b). These optimal values of $(d, \eta)$ are consistent with those determined in earlier studies using GNSS data (Sato et al 2011; Hu and Freymueller 2019). Additionally, the $\chi_{\min }^{2}$ value is close to the degree of freedom in the case of our grid search calculation $(=6-2=4)$, and this indicates that our calculation is statistically plausible.

Here, we define the error range of $\chi^{2}$ as $\chi^{2} \leq \chi_{\min }^{2}+\Delta \chi^{2}$, where $\Delta \chi^{2}$ indicates the $68.27 \%$ confidence level in the chi-squared test. Since $\Delta \chi^{2}=2.30$ for two variables, the error range of $\chi^{2}$ is calculated to be $3.39+2.30=5.69$ (green line in Figure $7 \mathrm{~b}$ ), and the resultant error ranges for $(d, \eta)$ are obtained 
as $53 \leq d \leq 58 \mathrm{~km}$ and $1.0 \times 10^{19} \leq \eta \leq 1.5 \times 10^{19} \mathrm{~Pa}$ s. Our error ranges are smaller than those of Hu and Freymueller (2019) by about 67 and $83 \%$ for $d$ and $\eta$, respectively. Their error ranges may be enlarged because one more parameter (i.e., asthenospheric thickness) was determined using their grid search analysis. These results indicate that absolute gravimetry, as well as GNSS observations (Sato et al 2011; Hu and Freymueller 2019) can precisely determine rheological parameters for the uppermost layers of the Earth.

\section{The behavior of Love numbers for RPIM and LPIM}

Figure 8 indicates the behavior of the Green's function obtained using the optimum rheological model for RPIM and LPIM (red cross in Fig. 7b). This figure is similar to Figure 6, showing the Love numbers of the VM5a model for GPIM, however, the ranges of the vertical axes in panels (a), (b), (d), and (e) differ between the two figures. Both $-\dot{\delta}_{n}(2 \mathrm{kyr})$ and $\dot{h}_{n}(2 \mathrm{kyr})$ reach maxima at $n=38$ (blue lines in Fig. $8 \mathrm{a}$ and b), and their peak values are nearly the same as those obtained from the VM5a model (dashed gray lines in Fig. 8a and b). However, the peak values of $-\dot{\delta}_{n}(1 \mathrm{kyr})$ and $\dot{h}_{n}(1 \mathrm{kyr})$ from our optimum model (the cyan lines in Fig. 8a and b) are approximately 2.8 times greater than those from the VM5a model (red lines in Fig. 6a and b). These results indicate that the structure of the Earth of our optimum model responds to the past ice unloading of $\tau<2 \mathrm{kyr}$ more strongly than that of the VM5a model, and the same characteristics was observed in the panels of $-\dot{\delta}(\varphi, \tau)$ and $\dot{h}(\varphi, \tau)$ (Fig. $8 \mathrm{~d}$ and e, respectively). These characteristics are caused by the fact that the asthenospheric viscosity in our optimum model $\left(1.2 \times 10^{19}\right.$ $\mathrm{Pa} \mathrm{s})$ is about $1 / 40$ of that in the VM5a model $\left(5.0 \times 10^{20} \mathrm{~Pa} \mathrm{~s}\right)$, and consequently the relaxation time is much shorter in our model.

Contrarily, the ratio of $-\dot{\delta} / \dot{h}$ in our model is consistent with that in the VM5a model. For example, the asymptotic value of $-\dot{\delta}_{n}(\tau) / \dot{h}_{n}(\tau)$ at $n \rightarrow 180$ is $0.17-0.18 \mu \mathrm{Gal} / \mathrm{mm}$ in both models (Figs. $6 \mathrm{c}$ and 8c), although the ratio value diverges at $n>80$ in our model because of the division by $\dot{h}_{n}(\tau) \simeq 0$. Additionally, $-\dot{\delta}(0, \tau) / \dot{h}(0, \tau)$ is $0.16-0.17 \mu \mathrm{Gal} / \mathrm{mm}$, which is common for any time and structure model (Figs. 6f and 8f). These results imply that the ratio of gravity change to vertical deformation tends to be independent of the structure of the Earth, as discussed in the earlier studies (e.g., Wahr et al 1995; Fang and Hager 2001).

\section{Calculated gravity variation rates}

Figure 9 shows the calculated rates of gravity variation due to the effects of GPIM, RPIM, LPIM, and $\operatorname{PDIM}\left(\dot{g}_{v}^{G P I M}, \dot{g}_{v}^{R P I M}, \dot{g}_{v}^{L P I M}\right.$ and $\dot{g}_{e a}^{P D I M}$, respectively) at each gravity point. The mean of $\dot{g}_{v}^{G P I M}$ 
(purple bars) is approximately $-0.37 \mu \mathrm{Gal} / \mathrm{yr}$, which is smaller than both $\dot{g}_{v}^{R P I M}$ and $\dot{g}_{v}^{L P I M}$, and the difference in $\dot{g}_{v}^{G P I M}$ between the gravity points was also small (within approximately $0.10 \mu \mathrm{Gal}$ ). This is because the gravity points in SE-AK are located far from the Laurentide ice sheet, which had covered North America and Canada until about 14 kyr BP (Peltier et al 2015).

$\dot{g}_{v}^{L P I M}$ and $\dot{g}_{v}^{R P I M}$ (blue and cyan bars in Fig. 9) are the first and second largest ice melting effects at each gravity point and their averages $(-1.87$ and $-0.71 \mu \mathrm{Gal} / \mathrm{yr})$ are about 5 and 2 times greater than that of $\dot{g}_{v}^{G P I M}$, respectively. This is because a significant mass of glaciers has melted in the low-viscosity asthenosphere of SE-AK in the last 100 years. Particularly, $\dot{g}_{v}^{L P I M}$ at GBCL, RUSG, and HNSG is about twice as large as that at the other gravity points because three gravity points are located in the center of the Glacier Bay area, where the significant ice mass has rapidly melted since LIA.

In the PDIM effects, the contribution of $\dot{g}_{e}^{U A F 07}$ (orange bars in Fig. 9) is the largest at almost all gravity points, because UAF07's PDIM model covers SE-AK, in which we measured the AG values. $\dot{g}_{a}^{U A F 07}$ (pink bars) is positive at many gravity points because the glacier melting at a higher position relative to a gravity point leads to the weakening of the upward attraction force (i.e., the gravity increase). In particular, $\dot{g}_{a}^{U A F 07}$ at MGVC is about 10 times larger than its average at the other five gravity points, and the contribution of $\dot{g}_{a}^{U A F 07}$ is the greatest for all PDIM effects at MGVC only. In fact, the MGVC gravity point is located in the Mendenhall Glacier Visitor Center, and closest to glaciers of all the gravity points (Table 1). Green bars indicate the sum of the four PDIM effects (i.e., $\left.\dot{g}_{e a}^{P D I M}=\dot{g}_{e}^{U A F 07}+\dot{g}_{a}^{U A F 07}+\dot{g}_{e}^{U A F 05}+\dot{g}_{a}^{U A F 05}\right)$; whereas $\dot{g}_{e a}^{P D I M}$ is positive $(+0.66 \mu \mathrm{Gal} / \mathrm{yr})$ at MGVC due to the $\dot{g}_{a}^{U A F 07}$ 's contribution, and it is negative ( -1 to $\left.0 \mu \mathrm{Gal} / \mathrm{yr}\right)$ at the other AG points due to the contribution of $\dot{g}_{e}^{U A F 07}$.

Red triangles in Figure 9 show the sum of all effects calculated in this study $\left(\dot{g}_{c a l}=\dot{g}_{v}^{G P I M}+\dot{g}_{v}^{R P I M}+\right.$ $\left.\dot{g}_{v}^{L P I M}+\dot{g}_{e a}^{P D I M}\right) \cdot \dot{g}_{c a l}$ is larger (in absolute value) at gravity points close to Glacier Bay, such as RUSG, HNSG, and GBCL $(-4.65,-3.76$ and $-3.67 \mu \mathrm{Gal} / \mathrm{yr}$, respectively), and this feature is consistent with GNSS observations (Fig. 1). On the other hand, the difference in $\dot{g}_{c a l}$ between EGAN and MGVC $(-2.43$ and $-1.51 \mu \mathrm{Gal} / \mathrm{yr}$, respectively) is as large as approximately $1 \mu \mathrm{Gal} / \mathrm{yr}$, even though the two AG points are close together (approximately $6.5 \mathrm{~km}$ ) and have almost the same uplift rate (Fig. 1). The difference in $\dot{g}_{c a l}$ is nearly equal to that in $\dot{g}_{a}^{U A F 07}$ between the two gravity points, which indicates that the attraction effect of PDIM is strongly affected at MGVC. $\dot{g}_{c a l}$ is consistent with $\dot{g}_{o b s}$ (green circles) within the error range of $\dot{g}_{o b s}$ at all gravity points, and the root-mean-square (RMS) difference was calculated 
to be approximately $0.36 \mu \mathrm{Gal} / \mathrm{yr}$. According to the earlier studies, the RMS difference between $\dot{g}_{\text {obs }}$ (black circles; Sun et al 2010) and $\dot{g}_{\text {cal }}$ (blue triangles; Sato et al 2012) was approximately $1.06 \mu \mathrm{Gal} / \mathrm{yr}$. Hence, the RMS value in our results is approximately $60 \%$ smaller than that in an earlier study (Sun et al 2010; Sato et al 2012). In fact, Sato et al (2012) calculated $\dot{g}_{c a l}$ by using rheological parameters of Sato et al (2011). They determined the parameters from the vertical deformation rate of the GNSS time series (e.g., Larsen et al 2004), not from the gravity variation rate in the earlier study (Sun et al 2010). In contrast, we determined the optimal rheological parameters (Fig. 7) so as to conform $\dot{g}_{c a l}$ with $\dot{g}_{o b s}$ (Fig. 5), and hence, we naturally obtained the resultant $\dot{g}_{c a l}$ values with the smaller RMS residual. We would like to emphasize here that we succeeded in determining the rheological parameters robustly due to the accurate gravity variation rates observed from the long-term repeated AG measurements in SE-AK.

Our $\dot{g}_{c a l}$ values (red triangles) were significantly different from those of Sato et al (2012) (blue triangles) by approximately $1.5 \mu \mathrm{Gal} / \mathrm{yr}$ in the RMS, even though our rheological parameters agreed well with those by Sato et al (2011). The deviation of $\dot{g}_{c a l}$ may be caused by the incorrect sign of the attraction effect of the PDIM in the estimation of Sato et al (2012) as follows: We can calculate the attraction effect due to PDIM according to Sato et al (2012) as

$$
\dot{g}_{a}^{\text {Sato }}\left(\theta_{p}, \lambda_{p}\right)=\dot{g}^{\text {Sato }}\left(\theta_{p}, \lambda_{p}\right)-\beta_{B} \cdot \dot{u}_{e}^{\text {Sato }}\left(\theta_{p}, \lambda_{p}\right)
$$

where $\dot{g}^{\text {Sato }}$ indicates the sum of the gravity variation rate associated with PDIM (Table 8 in Sato et al 2012), $\dot{u}_{e}^{\text {Sato }}$ indicates the vertical deformation rate associated with the PDIM (Table 7 in Sato et al 2012), and $\beta_{B}$ indicates the Bouguer gravity gradient. When we use the value of $-0.22 \mu \mathrm{Gal} / \mathrm{mm}$ for $\beta_{B}, \dot{g}_{a}^{S a t o}$ is calculated to be -1.61 to $+0.25 \mu \mathrm{Gal} / \mathrm{yr}$, which is negatively correlated with our calculation result, i.e., $\dot{g}_{a}^{U A F 05}+\dot{g}_{a}^{U A F 07}(=-0.09$ to $+1.13 \mu \mathrm{Gal} / \mathrm{yr})$. In fact, if we correctly recalculate the total gravity variation rate of Sato et al (2012) by reversing the sign of $\dot{g}_{a}^{\text {Sato }}$, the recalculated rate (diamonds in Fig. 9) is found to be consistent with our $\dot{g}_{c a l}$ (red circles) within $0.3 \mu \mathrm{Gal} / \mathrm{yr}$ in the RMS.

\section{Comparison with ground deformation data}

To verify our rheological model in terms of ground deformation, we calculate the rate of vertical ground deformation (denoted by $\dot{u}_{c a l}$ ) at each GNSS station in SE-AK using our rheological model, and compare it with the observed rate of vertical ground deformation (denoted by $\dot{u}_{o b s}$ ). As for the observed deformation rate, we mainly used the results of the GNSS campaign observations conducted in the earlier studies 
(Larsen et al 2004, 2005; Sato et al 2011). We also downloaded the time series of three-dimensional coordinates observed at the other 11 continuous GNSS stations from websites of UNAVCO (Herring et al 2016) or Nevada Geodetic Laboratory (Blewitt et al 2018). We then determined the vertical deformation rate at each GNSS station by separating the vertical time series into linear, annual, and step components using the least-squares method. Figure 10d shows the vertical deformation rate observed at 99 GNSS stations in SE-AK $\left(\dot{u}_{o b s}\right)$, and the rate is greater than $0 \mathrm{~mm} / \mathrm{yr}$ for almost all GNSS stations, and it is greatest $(+35.0 \mathrm{~mm} / \mathrm{yr})$ at ANIT (Sato et al 2011). Figure 10a also shows the histogram of the $\dot{u}_{\text {obs }}$ 's distribution; the average and SD for $\dot{u}_{o b s}$ are obtained as $+17.47 \pm 8.17 \mathrm{~mm} / \mathrm{yr}$ (red and green lines).

In contrast, the vertical deformation rate can be calculated using the same procedure as for calculating the gravity variation rate (Eqs. 4 and 10). The vertical ground deformation due to the past/present glacial melting ( $\dot{u}_{v}$ and $\dot{u}_{e}$, respectively) can be expressed by the following equations (e.g., Peltier 1974):

$$
\begin{gathered}
\dot{u}_{v}\left(\theta_{p}, \lambda_{p}, t_{0}\right)=\rho_{i} \iint \Delta H_{i}(\theta, \lambda, s) \dot{G}_{u v}\left(\varphi, t_{0}-s\right) d S d s \\
\dot{G}_{u v}\left(\varphi, t_{0}-s\right)=\frac{R}{m_{e}} \sum_{n=0}^{n_{\max }} \dot{h}_{n}\left(t_{0}-s\right) P_{n}(\cos \varphi) \\
\dot{u}_{e}\left(\theta_{p}, \lambda_{p}\right)=\rho_{i} \iint \dot{H}_{i}(\theta, \lambda) G_{u e}(\varphi) d S, \\
\dot{u}_{c a l}=\dot{u}_{v}+\dot{u}_{e} \\
=\left(\dot{u}_{v}^{G P I M}+\dot{u}_{v}^{R P I M}+\dot{u}_{v}^{L P I M}\right)+\dot{u}_{e}^{P D I M} \\
=\left(\dot{u}_{v}^{G P I M}+\dot{u}_{v}^{R P I M}+\dot{u}_{v}^{L P I M}\right)+\left(\dot{u}_{e}^{U A F 07}+\dot{u}_{e}^{U A F 05}\right)
\end{gathered}
$$

where $R$ and $G_{u e}$ indicate the typical values of the radius of the Earth $(=6378.1 \mathrm{~km})$ and elastic Green's function for vertical deformation due to point loading, respectively. We calculated the viscoelastic component $\left(\dot{u}_{v}\right)$ using the time derivative of loading Love number $\left(\dot{h}_{n} ;\right.$ Fig. 8$)$ and the spatiotemporal variations of GPIM, RPIM and LPIM. We also calculated the elastic component $\left(\dot{u}_{e}\right)$ using the function $G_{u e}$ expected from the 1066A Earth model (Gilbert and Dziewonski 1975; Matsumoto et al 2001) and the spatial distributions of PDIM (composed of the UAF05 and UAF07 models). Figure 10e shows the sum of the calculated vertical deformation rates $\left(\dot{u}_{c a l}=\dot{u}_{e}+\dot{u}_{v}\right)$ at each GNSS station; $\dot{u}_{c a l}$ is large (up to $+29.50 \mathrm{~mm} / \mathrm{yr}$ ) in Glacier Bay, and its distribution is similar to that of $\dot{u}_{\text {obs }}$ (panel (d)). Figure 10b also shows the histogram of the $\dot{u}_{\text {cal }}$ 's distribution; Its average and SD are calculated to be $+17.68 \pm 7.48$ $\mathrm{mm} / \mathrm{yr}$ (red and green lines).

Fig. $10 \mathrm{c}$ and $10 \mathrm{f}$ show the histogram and spatial distribution of the residual $\left(\dot{u}_{o b s}-\dot{u}_{c a l}\right)$, respectively. 
The residual is normally distributed within $10 \mathrm{~mm} / \mathrm{yr}$ at all GNSS stations in SE-AK. Its average $(-0.21$ $\mathrm{mm} / \mathrm{yr})$ is almost equal to $0 \mathrm{~mm} / \mathrm{yr}$, and its SD $(4.20 \mathrm{~mm} / \mathrm{yr})$ is about twice smaller than that of $\dot{u}_{\text {obs }}$ (the panel (a)). In these respects, the vertical ground deformation calculated from our rheological model agrees with that observed in SE-AK. Note that the average and SD of $\dot{u}_{o b s}-\dot{u}_{c a l}$ in our result are almost the same as those in Sato et al (2011) $(-0.8 \pm 3.3 \mathrm{~mm} / \mathrm{yr})$, although they obtained rheological parameters from the observed vertical deformation data directly. However, we succeeded in reproducing the deformation data using our rheological model, which was independently determined from the AG data.

\section{Discussion}

\section{Ratio of gravity change to vertical displacement}

The ratio of gravity variation to vertical ground deformation was discussed in earlier studies, associated with ice melting history and rheological structure. Wahr et al (1995) found that the viscous part of $\dot{g} / \dot{u}$ becomes a constant of about $-0.154 \mu \mathrm{Gal} / \mathrm{mm}$, based on empirical tests using a GIA model for Greenland and Antarctica. Olsson et al (2015) also calculated the $\dot{g} / \dot{u}$ values for three GIA regions (Laurentia, Fennoscandia and the British Isles) using several rheological models, and showed that the ratio differs more between the regions than between the earth models within each region. However, in the case of SE-AK, the characteristics of the ratio value have not been quantitatively discussed, mainly because both $\dot{u}$ and $\dot{g}$ include the elastic part owing to the PDIM, and not only the viscoelastic part due to past ice melting. In fact, Sun et al (2010) failed to isolate the elastic and viscoelastic parts from the $\dot{g}$ values observed in SE-AK from 2006 to 2008, even though they utilized a quantity of $d \Delta / d t$ which is expected to be independent of the viscoelastic effect (see Wahr et al 1995). Therefore, we quantified the ratio values of gravity variation to vertical ground deformation in the SE-AK area using the observed/estimated values of $\dot{u}$ and $\dot{g}$.

Figure 11a shows the raw ratio value, which is obtained as the observed gravity variation rate of the observed vertical deformation rate for each pair of the AG point and the continuous GNSS station (Table $1)$ :

$$
r_{\text {raw }}^{o b s}=\frac{\dot{g}_{o b s}}{\dot{u}_{o b s}} .
$$

The average and SD of $r_{\text {raw }}^{o b s}$ are $-0.160 \pm 0.030 \mu \mathrm{Gal} / \mathrm{mm} ; r_{\text {raw }}^{o b s}$ at some gravity points deviates from the average value because the PDIM's attraction part contained in $\dot{g}_{o b s}$ differs between each gravity point. 
Figure 11b shows the ratio value after the attraction effect was removed from the gravity variation rate.

We refer to this ratio value as the total ratio (e.g., Olsson et al 2015):

$$
\begin{aligned}
& r_{t o t}^{o b s}=\frac{\dot{g}_{o b s}-\dot{g}_{a}^{P D I M}}{\dot{u}_{o b s}}, \\
& r_{t o t}^{c a l}=\frac{\dot{g}_{c a l}-\dot{g}_{a}^{P D I M}}{\dot{u}_{c a l}} .
\end{aligned}
$$

The observed total ratio ( $r_{\text {tot }}^{\text {obs }}$; green in Fig. 11b) averages to $-0.173 \mu \mathrm{Gal} / \mathrm{mm}$, which is greater (in absolute value) than the raw ratio $\left(r_{\text {raw }}^{\text {obs }}\right.$; Fig. 11a) by 0.013 . The SD of $r_{\text {tot }}^{o b s}$ is smaller than that of $r_{\text {raw }}^{o b s}$ by 0.004 ; particularly, the deviation of the ratio value at MGVC from its average decreased from 0.046 (Fig. 11a) to 0.004 (Fig. 11b) by correcting for the PDIM attraction effect. Moreover, the calculated total ratio $\left(r_{\text {tot }}^{c a l}\right.$; red in Fig. 11b) was averaged to be $-0.177 \pm 0.001 \mu \mathrm{Gal} / \mathrm{mm}$, which is consistent with $r_{\text {tot }}^{\text {obs }}$ within their error ranges. These results indicate that the attraction part due to the PDIM should be subtracted from the observed gravity data to obtain the $\dot{g} / \dot{u}$ 's ratio associated with GIA accurately. Figure 11c shows the viscous ratio value, i.e. the gravity variation rate to vertical deformation rate relating to past ice melting:

$$
\begin{aligned}
& r_{v}^{o b s}=\frac{\dot{g}_{o b s}-\dot{g}_{e a}^{P D I M}}{\dot{u}_{o b s}-\dot{u}_{e}^{P D I M}}, \\
& r_{v}^{c a l}=\frac{\dot{g}_{c a l}-\dot{g}_{e a}^{P D I M}}{\dot{u}_{c a l}-\dot{u}_{e}^{P D I M}}=\frac{\dot{g}_{v}^{G P I M}+\dot{g}_{v}^{R P I M}+\dot{g}_{v}^{L P I M}}{\dot{u}_{v}^{G P I M}+\dot{u}_{v}^{R P I M}+\dot{u}_{v}^{L P I M}} .
\end{aligned}
$$

The observed viscous ratio $\left(r_{v}^{\text {obs }}\right.$; green in Fig. 11c) averages to $-0.168 \mu \mathrm{Gal} / \mathrm{mm}$, which agrees with the average of the calculated viscous ratio $\left(r_{v}^{c a l}=-0.171 \mu \mathrm{Gal} / \mathrm{mm}\right.$; red line in Fig. 11c) within their error ranges. The absolute value of the viscous ratio in SE-AK was larger than that in other GIA regions $(-0.15$ to $-0.16 \mu \mathrm{Gal} / \mathrm{mm}$; Wahr et al 1995 ; Olsson et al 2015) because the glacier has melted in SE-AK after LIA, which is more recent than in other regions. In fact, the Green's function for LPIM and RPIM indicates that the ratio value becomes greater (in absolute) for a younger melting event in the vicinity of a gravity point (see the range of $0 \leq \phi \leq 2$ deg in Fig. 8f).

Figure 11d shows the elastic ratio value, obtained as the gravity change to the vertical deformation associated with GIA due to PDIM only:

$$
\begin{aligned}
& r_{e}^{o b s}=\frac{\dot{g}_{o b s}-\dot{g}_{v}-\dot{g}_{a}^{P D I M}}{\dot{u}_{o b s}-\dot{u}_{v}}, \\
& r_{e}^{c a l}=\frac{\dot{g}_{e}^{P D I M}}{\dot{u}_{e}^{P D I M}} .
\end{aligned}
$$

${ }_{441}$ The observed elastic ratio ( $r_{e}^{o b s}$; green in Fig. 11d) averages to $-0.087 \pm 0.145 \mu \mathrm{Gal} / \mathrm{mm}$, which significantly differs from the calculated elastic ratio $\left(r_{e}^{c a l}=-0.222 \pm 0.003 \mu \mathrm{Gal} / \mathrm{mm}\right.$; red in Fig. 11d). 
Particularly, $r_{e}^{o b s}$ at three AG points (RUSG, HNSG, and BRMC) largely deviate from $r_{e}^{c a l}$, and the same characteristics can be seen for these AG points in the other panels (Fig. 11a-c). In the case of RUSG and BRMC, the cause of the inconsistency between $r_{e}^{o b s}$ and $r_{e}^{c a l}$ may be related to a systematic error in the observed gravity variation rate. As shown in Figure $9, \dot{g}_{\text {obs }}$ deviates from $\dot{g}_{c a l}$ more at RUSG and BRMC than at the other AG points because the available AG data were fewer at RUSG and BRMC (Fig. 5), and hence, more AG data should be measured to determine the rate of gravity change more accurately. In the case of HNSG, the cause of the inconsistency between $r_{e}^{o b s}$ and $r_{e}^{c a l}$ may be due to the difference in location between the AG and GNSS points. We used the GNSS data collected at AB44 in Skagway (35 km north of HNSG in Haines) because no continuous GNSS station has been installed in Haines thus far. The installation of a continuous GNSS station around HNSG should be considered in a future study to compare ground displacement with gravity change directly. The observed elastic ratios at the other three AG points (EGAN, MGVC and GBCL) average to $-0.207 \pm 0.049 \mu \mathrm{Gal} / \mathrm{mm}$, which agrees with the average of $r_{e}^{c a l}$ within their error ranges.

The difference between $r_{v}$ and $r_{e}$ (Fig. 11c-d) can be explained mainly by the density value for each subsurface layer associated with GIA. Generally, the ratio of the gravity variation to the vertical displacement can be approximately calculated by the Bouguer's gravity gradient:

$$
\beta_{B}=\beta_{F}+2 \pi \rho G
$$

where the first and second terms indicate the free-air gradient $(=-0.3086 \mu \mathrm{Gal} / \mathrm{mm}$ as a typical value; Garland 1965) and the gravity change caused by a unit-thickness infinite plane with a density value of $\rho$, respectively. The viscoelastic part of the GIA can be interpreted as thickening of the upper-mantle thickness, and thus, the $\beta_{B}$ value with a typical upper-mantle density of $3300 \mathrm{~kg} / \mathrm{m}^{3}$ is calculated to be $-0.17 \mu \mathrm{Gal} / \mathrm{mm}$, which is exactly consistent with $r_{v}$ (Fig. 11c). In contrast, $r_{e}^{c a l}(=-0.222 \mu \mathrm{Gal} / \mathrm{mm})$ is approximately $10 \%$ greater (in absolute) than $\beta_{B}(=-0.197 \mu \mathrm{Gal} / \mathrm{mm})$ when using a typical crustal density of $2670 \mathrm{~kg} / \mathrm{m}^{3}$ for $\rho$ because $r_{e}^{c a l}$ was calculated by considering the spatial variation in the medium density (see Farrell 1972).

The values of $r_{v}^{c a l}$ and $r_{e}^{c a l}$ may be utilized in separating the elastic/viscoelastic parts from the geodetic data acquired in SE-AK. If we assume that the attraction effect due to PDIM is negligible in the observed gravity change, the observed crustal deformation $\left(\dot{u}_{o b s}\right)$ and gravity change $\left(\dot{g}_{o b s}\right)$ can be written as (e.g., 
Sugawara 2011)

$$
\begin{aligned}
& \dot{u}_{o b s}=\dot{u}_{v}^{e m p}+\dot{u}_{e}^{e m p}, \\
& \dot{g}_{o b s}=\dot{g}_{v}^{e m p}+\dot{g}_{e}^{e m p}=r_{v}^{c a l} \cdot \dot{u}_{v}^{e m p}+r_{e}^{c a l} \cdot \dot{u}_{e}^{e m p},
\end{aligned}
$$

where each term with superscript emp indicates the value separated by this empirical method. When we substitute the observed values at GBCL (i.e., $+21.23 \mathrm{~mm} / \mathrm{yr}$ and $-3.92 \mu \mathrm{Gal} / \mathrm{yr}$ ) into these equations, $\dot{u}_{v}^{e m p}$ and $\dot{u}_{e}^{e m p}$ are estimated to be +15.53 and $+5.71 \mathrm{~mm} / \mathrm{yr}$, respectively, and the value of $\dot{u}_{v}^{e m p}$ agrees well with that obtained from our numerical calculation $(=+18.87 \mathrm{~mm} / \mathrm{yr})$. However, in the case of MGVC, the value of $\dot{u}_{e}^{e m p}(=-20.28 \mathrm{~mm} / \mathrm{yr})$ differs from the calculation result $\left(r_{v}^{c a l}=+2.08 \mathrm{~mm} / \mathrm{yr}\right)$ in terms of the sign and absolute value because the attraction effect due to PDIM cannot be ignored at MGVC (Fig. 9). In these respects, this empirical method is valid only when the PDIM attraction effect is small.

\section{The effect of acceleration in glacier melting}

Earlier studies discussed the possibility of acceleration in glacier melting in the SE-AK region (e.g., Arendt et al 2002). For example, Hu and Freymueller (2019) found that the rheological parameters for SE-AK were estimated differently if they used the GNSS time series observed in SE-AK during 1992-2003 or 2003-2012. They mentioned that the estimation results were distorted by the time variation in glacier melting rate during the analysis period from 1992 to 2012, and considered scale factors for a PDIM model (Berthier et al 2010) so as to obtain the consistent rheological parameters regardless of analysis periods. The scale factors were calculated as 1.8 for $1992-2003$ and 2.2 for $2003-2012$, and they concluded that the recent PDIM rate duplicated relative to the PDIM model which is based on the ice elevation change from the 1960s to the 2000s (Berthier et al 2010), and the ice melting rate accelerated by approximately $20 \%$ in and after 2003.

We examine the dependence of the rheological parameters to the PDIM rate by changing a scale factor for the PDIM models (Arendt et al 2002; Larsen et al 2005, 2007) in the grid search calculation. Figure 12 shows the variations in the optimal parameters for $(d, \eta)$ and their error ranges when we vary the scale factor for PDIM by 0.1 between 0.5 and 2.0. The optimal value of the lithospheric thickness $(d$; red line) changed by just $3 \mathrm{~km}(d=54-57 \mathrm{~km})$ to the scale factor, and the optimal value of the upper mantle viscosity $(\eta)$ with a 1.0's scale factor (the blue star) is located within the overall error range when the scale factor is varied from 0.5 to 2.0 . In these respects, the model parameters were robustly determined 
in this study to be $d=55 \mathrm{~km}$ and $\eta=1.2 \times 10^{19} \mathrm{~Pa} \mathrm{~s}$ (the stars in Fig. 12; see Fig. 7 ), as long as we used absolute gravity data (Fig. 5) for estimation of the model parameters. In fact, most of our AG points are insensitive to the PDIM effect because the gravity change due to PDIM was calculated to be smaller than those due to LPIM and RPIM (Fig. 9). Although the PDIM effect was largest at MGVC (Mendenhall Glacier Visitor Center) in all of the AG points, the AG variation rate was obtained with the lowest precision at MGVC (Fig. 5). Therefore, its weight was the smallest during our parameter estimation (Eq. 8). The scale factor for PDIM may be determined from the AG data directly if we repeatedly measure AG values at MGVC with high accuracy in the future.

The upper mantle viscosity $(\eta)$ slightly increases with an increase in the PDIM scale factor (Fig. 12) associated with the ice melting history in SE-AK as follows: when the PDIM scale factor is greater, the gravity variation rate due to PDIM (the green bars in Fig. 9) is also calculated to be greater in absolute value. The contributions of RPIM and LPIM (blue and cyan bars in Fig. 9, respectively) need to get smaller at most of the AG points to reconcile the sum of the ice-melting effects with the observed AG variation (red triangles and green circles in Fig. 9). Since RPIM and LPIM occurred in the SE-AK area after the end of the LIA in the 19-20th centuries (Larsen 2003; Larsen et al 2005), which is more recent than GPIM, $\eta$ 's increase leads to an increase in the time delay between the ice melting and the viscous ground uplift, and the resultant decrease in the present AG variation due to the RPIM and LPIM.

\section{Conclusions}

To understand the spatiotemporal gravity variation associated with past/present ice melting, we measured the AG values at six gravity points in SE-AK in 2012, 2013, and 2015, and reprocessed the new AG data in the common procedure with the past AG data obtained in 2006-2008 (Sun et al 2010; Sato et al 2012). After correcting the effects of hydrological and coseismic gravity changes from the reprocessed AG values, we updated the gravity variation rate at the gravity points in SE-AK, using the AG time series from 2006 to 2015. The updated gravity variation rate ranged from $-2.05 \mu \mathrm{Gal} / \mathrm{yr}$ (at MGVC, the gravity point located in the Mendengall Glacier Visitor Center) to $-4.40 \mu \mathrm{Gal} / \mathrm{yr}$ (at RUSG, the gravity point located on Russell Island), and averaged to be $-3.25 \mu \mathrm{Gal} / \mathrm{yr}$. The standard deviation of the updated rate is much smaller than that in the previous study (Sun et al 2010) at most of the gravity points because our new AG measurements in 2012-2015 extended the data period from 3 years (Sun et al 2010) to 10 years. We then reproduced the observed AG variation rate, by numerically convolving the past/present-day ice 
melting models (Arendt et al 2002; Larsen et al 2004, 2005, 2007; Peltier et al 2015) and Green's functions for viscoelastic/elastic loading deformations (Farrell 1972; Peltier 1974). To reproduce the observed AG variation rate, we searched for the optimal rheological parameters of the lithospheric thickness $(d)$ and upper mantle viscosity $(\eta)$ for the significant ice melting event that occurred in SE-AK at the end of the LIA. The optimal rheological parameters were determined to be $d=55_{-2}^{+3} \mathrm{~km}$ and $\eta=\left(1.2_{-0.2}^{+0.3}\right) \times 10^{19}$ Pa s, which were both consistent with those obtained by earlier studies using GNSS data (Sato et al 2011; Hu and Freymueller 2019). The error ranges of our optimal rheological parameters were as small as those in the most recent GNSS study (Hu and Freymueller 2019); this result indicates that the rheological parameters under SE-AK can be precisely determined from the AG data, as well as from the GNSS data (e.g., Sato et al 2011; Hu and Freymueller 2019). Additionally, the differences between observation and calculation were estimated to be $0.36 \mu \mathrm{Gal} / \mathrm{yr}$ for the AG time series and $0.21 \mathrm{~mm} / \mathrm{yr}$ for the GNSS time series, which are smaller than those in the earlier studies (e.g., Sato et al 2011, 2012). In this respect, we succeeded in obtaining a robust rheological model for SE-AK, which agrees with both of gravity and crustal deformation data.

We utilized the observed/calculated AG variation rates to estimate the ratio of the AG variation to the vertical displacement at the gravity points in SE-AK. The viscous ratio $\left(r_{v}\right)$ was $-0.168 \mu \mathrm{Gal} / \mathrm{mm}$ from the observed data and $-0.171 \mu \mathrm{Gal} / \mathrm{mm}$ from the calculated result. These viscous ratio values for SEAK are greater (in absolute) than those for other GIA regions ( -0.15 to $-0.16 \mu \mathrm{Gal} / \mathrm{mm}$; Wahr et al 1995; Olsson et al 2015) because of the difference in the ice-melting history; significant mass of glacier melted in SE-AK after LIA, which is more recent than in the other regions. The elastic ratio $\left(r_{e}\right)$ was also obtained as $-0.222 \mu \mathrm{Gal} / \mathrm{mm}$ from our numerical calculation. This value is greater (in absolute) than the typical Bouguer's gravity gradient $(-0.197 \mu \mathrm{Gal} / \mathrm{mm})$ due to the effect of the spatiotemporal variation in medium density (e.g., Farrell 1972).

We finally examined the dependence of the optimal rheological parameters to the PDIM rate by changing a scale factor for the PDIM models (e.g., Hu and Freymueller 2019). However, we found that the optimal parameters do not change with the variation in the scale factor. Specifically, the acceleration of PDIM cannot be confirmed from our AG dataset (Table 2), because the gravity changes are only weakly sensitive to the PDIM component. Even though the MGVC gravity point is close to Mendenhall Glacier, the AG values at MGVC are not sensitive to PDIM because of the large SD of the AG variation rate associated with the relocation the AG point between 2008 and 2012. To validate the acceleration of PDIM in the 
SE-AK region (Hu and Freymueller 2019) based on gravimetry, the temporal AG variation should be monitored at the six AG points by measuring the AG values repeatedly in the future.

\section{List of abbreviations}

AG: absolute gravity

BP: before present

FY: fiscal year

GIA: glacial isostatic adjustment

GNSS: Global Navigation Satellite System

GLDAS: Global Land Data Assimilation System

GPIM: global past ice melting

IERS: International Earth Rotation Service

ISEA: International Geodetic Measurements in Southeast Alaska

LIA: Little Ice Age

LPIM: local past ice melting

PDIM: preset-day ice melting

PREM: Preliminary Reference Earth Model

RMS: root mean square

RPIM: regional past ice melting

SD: standard deviation

SE-AK: Southeast Alaska

SNOTEL: Snow Telemetry

\section{Declarations}

\section{Availability of data and materials}

The SNOTEL snow accumulation data was provided by NRCS (https://www.wcc.nrcs.usda.gov/ snow/). The Green's functions for elastic loading deformation based on the 1066A Earth model are available in the GOTIC2's software for computing oceanic tidal loading effects (Matsumoto et al 2001; https://www.miz.nao.ac.jp/staffs/nao99/). The ETOPO1's digital elevation model is provided by NOAA (https://www.ngdc.noaa.gov/mgg/global/). The GLDAS-2.1's global hydrological model 
is provided by the NASA Goddard Earth Science Data and Information Services Center (https: //ldas.gsfc.nasa.gov/gldas). The slip distribution data of the Haida Gwaii and Craig Earthquakes (Lay et al 2013) are provided by Thorne Lay (University of California Santa Cruz). The GNSS data sets are provided by UNAVCO (https://www.unavco.org/data/gps-gnss/gps-gnss.html) and Nevada Geodetic Laboratory (http://geodesy.unr.edu/magnet.php). The ICE6G_C's ice melting model is available from the web page of W. R. Peltier (https://www.atmosp.physics.utoronto.ca/ peltier/ data.php). The ice melting models of RPIM, LPIM, and PDIM are available from the corresponding author, C. F. Larsen (University of Alaska Fairbanks).

\section{Competing interests}

The authors declare that they have no competing interests.

\section{Funding}

This study was supported by JSPS KAKENHI Grant Numbers JP17253003 and JP23253003 from the Japanese Ministry of Education, Culture, Sports, Science and Technology.

\section{Authors' contributions}

TK, SM, HH, and YO measured the AG values in SE-AK from 2012 to 2015, and TK reprocessed the AG data collected from 2006 to 2015. KN analyzed the AG/GNSS data and constructed a GIA model for SE-AK. SM and JF provided overall supervision for this study, and YF provided technical advice for this study. KN drafted most of the manuscript, while TK drafted the manuscript of the section titled "Absolute gravity data". All authors read and approved the final manuscript.

\section{Authors' information}

KN and TK, Graduate School of Science, Kyoto University, Sakyo-ku, Kyoto 606-8502, Japan YF, Graduate School of Science, Kyoto University, Sakyo-ku, Kyoto 606-8502, Japan; Now at National Institute of Polar Research, Tachikawa, Tokyo 190-8518, Japan

SM and YO, Research Center for Prediction of Earthquakes and Volcanic Eruptions, Graduate School of Science, Tohoku University

HH, Institute for Cosmic Ray Research, the University of Tokyo, Kashiwa, Chiba 277-8582, Japan 
JF, Geophysical Institute, University of Alaska Fairbanks, 2156 Koyukuk Drive, Fairbanks, AK 99775, USA; Now at Department of Earth and Environmental Sciences, Michigan State University, East Lansing, MI 48824, USA

\section{Acknowledgments}

We are grateful to Tadahiro Sato (National Astronomical Observatory of Japan; Tohoku University), who passed away on 23 May 2021 at the age of 76, helped us measure AG values in SE-AK and provided us with technical information to calculate the GIA response. We thank Max Kaufman (University of Alaska Fairbanks), Sam Herreid (Northumbria University), Kimberly DeGrandpre (Michigan State University), Yuning Fu (Bowling Green State University), and Gabrielle Vance (University of Alaska Southeast) for helping us measure the AG values in SE-AK in 2012-2015. GMT (version 5.1.1) (Wessel et al 2013) was used to create some figures in this study.

\section{Appendix A. Description of absolute gravity points}

Sun et al (2010) described the details of the following five AG points: EGAN (Egan Library, University of Alaska Southeast), MGVC (Mendenhall Glacier Visitor Center), GBCL (Gustavus Bartlett Cove), RUSG (Russell Island) and HNSG (Haines Fairground). In this appendix, we describe the detailed location of the MGVC gravity point (Fig. A1) because we compellingly relocated the gravity point in June 2012 due to an exhibit installed on the old gravity point by the visitor center. We also show the detailed location of BRMC (Blanchard Road Maintenance Compound) gravity point (Fig. A2), because earlier studies (Sun et al 2010; Sato et al 2012) did not describe its detailed location. Although Sun et al (2010) did not collect any AG data at BRMC in June 2006, Sato et al (2012) measured the AG values at BRMC in June of 2007 and 2008 as part of the first ISEA project, and compared the AG values with those obtained at the same AG point in 1987 (Sasagawa et al 1989).

\section{Endnotes}

\section{References}

Amante C, Eakins B (2009) ETOPO1 1 arc-minute global relief model: procedures, data sources and analysis. NOAA technical memorandum NESDIS NGDC-24. National Geophysical Data Center, 
Arendt AA, Echelmeyer KA, Harrison WD, Lingle CS, Valentine VB (2002) Rapid wastage of Alaska glaciers and their contribution to rising sea level. Science 297(5580):382-386,

DOI $10.1126 /$ science.1072497

Berthier E, Schiefer E, Clarke GK, Menounos B, Rémy F (2010) Contribution of Alaskan glaciers to sea-level rise derived from satellite imagery. Nature Geoscience 3(2):92-95, DOI 10.1038/ngeo737

Blewitt G, Hammond WC, Kreemer C (2018) Harnessing the GPS data explosion for interdisciplinary science. Eos 99(10.1029):485, DOI 10.1029/2018EO104623

Dziewonski AM, Anderson DL (1981) Preliminary reference Earth model. Physics of the earth and planetary interiors 25(4):297-356, DOI 10.1016/0031-9201(81)90046-7

Ekman M, Mäkinen J (1996) Recent postglacial rebound, gravity change and mantle flow in Fennoscandia. Geophysical Journal International 126(1):229-234,

DOI 10.1111/j.1365-246X.1996.tb05281.x

Elliott JL, Larsen CF, Freymueller JT, Motyka RJ (2010) Tectonic block motion and glacial isostatic adjustment in southeast Alaska and adjacent Canada constrained by GPS measurements. Journal of Geophysical Research: Solid Earth 115(B9), DOI 10.1029/2009JB007139

Fang M, Hager BH (2001) Vertical deformation and absolute gravity. Geophysical Journal International 146(2):539-548, DOI 10.1046/j.0956-540x.2001.01483.x

Farrell W (1972) Deformation of the Earth by surface loads. Reviews of Geophysics 10(3):761-797, DOI 10.1029/RG010i003p00761

Garland GD (1965) The Earth's Shape and Gravity. Pergamon Press

Gilbert F, Dziewonski AM (1975) An application of normal mode theory to the retrieval of structural parameters and source mechanisms from seismic spectra. Philosophical Transactions of the Royal Society of London Series A, Mathematical and Physical Sciences 278(1280):187-269, DOI 10.1098/rsta.1975.0025

Herring TA, Melbourne TI, Murray MH, Floyd MA, Szeliga WM, King RW, Phillips DA, Puskas CM, Santillan M, Wang L (2016) Plate Boundary Observatory and related networks: GPS data analysis methods and geodetic products. Reviews of Geophysics 54(4):759-808, DOI 10.1002/2016RG000529x Hu Y, Freymueller JT (2019) Geodetic observations of time-variable glacial isostatic adjustment in Southeast Alaska and its implications for Earth rheology. Journal of Geophysical Research: Solid Earth 124(9):9870-9889, DOI 10.1029/2018JB017028 
Huss M (2013) Density assumptions for converting geodetic glacier volume change to mass change. The

Cryosphere 7(3):877-887, DOI 10.5194/tc-7-877-2013,2013

Inazu D, Sato T, Miura S, Ohta Y, Nakamura K, Fujimoto H, Larsen CF, Higuchi T (2009) Accurate ocean tide modeling in southeast Alaska and large tidal dissipation around Glacier Bay. Journal of oceanography 65(3):335-347, DOI 10.1007/s10872-009-0031-y

James TS, Ivins ER (1998) Predictions of Antarctic crustal motions driven by present-day ice sheet evolution and by isostatic memory of the Last Glacial Maximum. Journal of Geophysical Research: Solid Earth 103(B3):4993-5017, DOI 10.1029/97JB03539

Larsen CF (2003) Rapid uplift of southern alaska caused by recent ice loss. PhD thesis, University of Alaska Fairbanks, http://fairweather.alaska.edu/chris/thesis.pdf, (Accessed on 30 June 2021)

Larsen CF, Motyka RJ, Freymueller JT, Echelmeyer KA, Ivins ER (2004) Rapid uplift of southern Alaska caused by recent ice loss. Geophysical Journal International 158(3):1118-1133, DOI 10.1111/j.1365-246X.2004.02356.x

Larsen CF, Motyka RJ, Freymueller JT, Echelmeyer KA, Ivins ER (2005) Rapid viscoelastic uplift in southeast Alaska caused by post-Little Ice Age glacial retreat. Earth and planetary Science letters 237(3-4):548-560, DOI 10.1016/j.epsl.2005.06.032

Larsen CF, Motyka RJ, Arendt AA, Echelmeyer KA, Geissler PE (2007) Glacier changes in southeast Alaska and northwest British Columbia and contribution to sea level rise. Journal of Geophysical Research: Earth Surface 112(F1), DOI 10.1029/2006JF000586

Lay T, Ye L, Kanamori H, Yamazaki Y, Cheung KF, Kwong K, Koper KD (2013) The October 28, 2012 Mw 7.8 Haida Gwaii underthrusting earthquake and tsunami: Slip partitioning along the Queen Charlotte Fault transpressional plate boundary. Earth and Planetary Science Letters 375:57-70, DOI 10.1016/j.epsl.2013.05.005

Matsumoto K, Sato T, Takanezawa T, Ooe M (2001) GOTIC2: A program for computation of oceanic tidal loading effect. Journal of the Geodetic Society of Japan 47(1):243-248,

DOI 10.11366/sokuchi1954.47.243

Micro-g LaCoste (2006) FG5 Absolute Gravimeter User's Manual. http://microglacoste.com/pdf/FG5Manual2007.pdf, (Accessed on 30 June 2021)

Micro-g LaCoste (2012) g9 User's Manual. http://microglacoste.com/pdf/g9Help.pdf, (Accessed on 
30 June 2021)

Nykolaishen L, Dragert H, Wang K, James TS, Schmidt M (2015) GPS observations of crustal deformation associated with the $2012 \mathrm{Mw} 7.8$ Haida Gwaii earthquake. Bulletin of the Seismological Society of America 105(2B):1241-1252, DOI 10.1785/0120140177

Okubo S (1992) Gravity and potential changes due to shear and tensile faults in a half-space. Journal of Geophysical Research: Solid Earth 97(B5):7137-7144, DOI 10.1029/92JB00178

Olsson PA, Milne G, Scherneck HG, Ågren J (2015) The relation between gravity rate of change and vertical displacement in previously glaciated areas. Journal of Geodynamics 83:76-84, DOI 10.1016/j.jog.2014.09.011

Peltier W (1974) The impulse response of a Maxwell Earth. Reviews of Geophysics 12(4):649-669, DOI 10.1029/RG012i004p00649

Peltier WR, Argus D, Drummond R (2015) Space geodesy constrains ice age terminal deglaciation: The global ICE-6G_C (VM5a) model. Journal of Geophysical Research: Solid Earth 120(1):450-487, DOI 10.1002/2014JB011176

Richards MA, Lenardic A (2018) The Cathles parameter (Ct): a geodynamic definition of the asthenosphere and implications for the nature of plate tectonics. Geochemistry, Geophysics, Geosystems 19(12):4858-4875, DOI 10.1029/2018GC007664

Rodell M, Houser P, Jambor U, Gottschalck J, Mitchell K, Meng CJ, Arsenault K, Cosgrove B, Radakovich J, Bosilovich M, et al (2004) The global land data assimilation system. Bulletin of the American Meteorological Society 85(3):381-394, DOI 10.1175/BAMS-85-3-381

Sasagawa GS, Zumberge MA, Stevenson JM, Lautzenhiser T, Wirtz J, Ander ME (1989) The 1987 Southeastern Alaska gravity calibration range: Absolute and relative gravity measurements. Journal of Geophysical Research: Solid Earth 94(B6):7661-7666, DOI 10.1029/JB094iB06p07661

Sato T, Larsen CF, Miura S, Ohta Y, Fujimoto H, Sun W, Motyka RJ, Freymueller JT (2011) Reevaluation of the viscoelastic and elastic responses to the past and present-day ice changes in Southeast Alaska. Tectonophysics 511(3-4):79-88, DOI 10.1016/j.tecto.2010.05.009

Sato T, Miura S, Sun W, Sugano T, Freymueller JT, Larsen CF, Ohta Y, Fujimoto H, Inazu D, Motyka RJ (2012) Gravity and uplift rates observed in southeast Alaska and their comparison with GIA model predictions. Journal of Geophysical Research: Solid Earth 117(B1), 
Spada G (2008) ALMA, a Fortran program for computing the viscoelastic love numbers of a spherically symmetric planet. Computers \& Geosciences 34(6):667-687, DOI 10.1016/j.cageo.2007.12.001

Sugawara Y (2011) Gravity measurements with the portable absolute gravimeter FG5 at Antarctica (IV). Bull Geospat Info Authority Japan 121:9-19, in Japanese

Sun W, Miura S, Sato T, Sugano T, Freymueller J, Kaufman M, Larsen C, Cross R, Inazu D (2010)

Gravity measurements in southeastern Alaska reveal negative gravity rate of change caused by glacial isostatic adjustment. Journal of Geophysical Research: Solid Earth 115(B12),

DOI 10.1029/2009JB007194

Timmen L, Wenzel HG (1995) Worldwide synthetic gravity tide parameters. In: Gravity and geoid, Springer, pp 92-101, DOI 10.1007/978-3-642-79721-7_11

Wahr J, DaZhong H, Trupin A (1995) Predictions of vertical uplift caused by changing polar ice volumes on a viscoelastic Earth. Geophysical Research Letters 22(8):977-980, DOI 10.1029/94GL02840

Wessel P, Smith WH, Scharroo R, Luis J, Wobbe F (2013) Generic mapping tools: improved version released. Eos, Transactions American Geophysical Union 94(45):409-410

\section{Tables}


Table 1. : Parameters of AG points and their nearest GNSS stations.

\begin{tabular}{|c|c|c|c|c|c|c|}
\hline$A G$ point & & & & & & \\
\hline Name & EGAN & MGVC & GBCL & RUSG & HNSG & BRMC \\
\hline Latitude [deg] & 58.38534 & 58.41736 & 58.45462 & 58.90639 & 59.23186 & 60.00183 \\
\hline Longitude [deg] & -134.64002 & -134.54617 & -135.87488 & -136.78908 & -135.45977 & -136.85511 \\
\hline Elevation $[\mathrm{m}]$ & 38.0 & 8.0 & 21.0 & 10.0 & 21.0 & 840.0 \\
\hline Nominal air pressure $[\mathrm{hPa}]$ & 1008.69 & 1012.29 & 1010.73 & 1012.05 & 1010.73 & 916.33 \\
\hline Gravity gradient $[\mu \mathrm{Gal} / \mathrm{cm}]$ & -2.734 & $\begin{array}{l}-2.045^{\# 1} \\
-2.364^{\# 2}\end{array}$ & -3.099 & -3.468 & -3.093 & -2.910 \\
\hline Distance to nearest glacier $\# 3[\mathrm{~km}]$ & 7.37 & 1.46 & 30.82 & 6.81 & 11.56 & 31.09 \\
\hline \multicolumn{7}{|l|}{ Continuous GNSS station } \\
\hline Name & AB50 & AB50 & GUS0\#4 & QUIC & $\mathrm{AB} 44$ & $\mathrm{MDFC}$ \\
\hline Data analyst & UNAVCO & UNAVCO & UNAVCO & NGL $\# 5$ & UNAVCO & NGL \\
\hline Latitude [deg] & 58.41678 & 58.41678 & 58.41776 & 58.90856 & 59.52804 & 60.12182 \\
\hline Longitude [deg] & -134.54530 & -134.54530 & -135.69705 & -136.58682 & -135.22830 & -136.95833 \\
\hline Elipsoidal height $[\mathrm{m}]$ & 51.6 & 51.6 & 19.9 & 23.8 & 304.2 & 897.0 \\
\hline Distance from AG point $[\mathrm{km}]$ & 6.53 & 0.08 & 11.13 & 11.61 & 35.42 & 14.51 \\
\hline
\end{tabular}

\#1 Gravity gradient value at the old AG point in 2006-2008.

\#2 Gravity gradient value at the new AG point in 2012-2015.

\#3 The 3-D distances from each AG point to the nearest glacier using the PDIM model (UAF07, Larsen et al 2007).

\#4 Because the GNSS station of GUS2 was replaced to GUS6 on 20 June 2008, we named a hypothetical GNSS station GUS0 and created its continuous time series from those collected at GUS2 and GUS6 by removing coordinate steps due to the relocation of the GNSS station.

\#5 Nevada Geodetic Laboratory (NGL). 
Table 2. : Reprocessed AG values at gravity points in SE-AK.\#1

\begin{tabular}{|c|c|c|c|c|c|c|c|c|}
\hline AG point & Year & Dates & $\begin{array}{c}\text { AG value } \\
{[\mu \mathrm{Gal}]}\end{array}$ & $\begin{array}{c}\mathrm{SD} \\
{[\mu \mathrm{Gal}]}\end{array}$ & $\begin{array}{c}\mathrm{SE} \\
{[\mu \mathrm{Gal}]}\end{array}$ & $\begin{array}{c}\mathrm{UC} \\
{[\mu \mathrm{Gal}]}\end{array}$ & $N_{s}$ & $N_{d}$ \\
\hline \multirow[t]{6}{*}{ EGAN } & 2006 & Jun14-Jun16 & 981760220.08 & 1.56 & 0.17 & 2.18 & 89 & 8834 \\
\hline & 2007 & May31-Jun02 & 981760219.75 & 1.10 & 0.14 & 2.12 & 66 & 6568 \\
\hline & 2008 & May30-Jun01 & 981760211.63 & 1.24 & 0.13 & 2.12 & 95 & 9437 \\
\hline & 2012 & Jun13-Jun15 & 981760207.25 & 1.70 & 0.13 & 2.12 & 169 & 16760 \\
\hline & 2013 & Jun10-Jun 12 & 981760193.50 & 2.03 & 0.19 & 2.18 & 118 & 14082 \\
\hline & 2015 & May30-Jun02 & 981760194.66 & 2.52 & 0.22 & 2.13 & 133 & 13110 \\
\hline \multirow[t]{6}{*}{ MGVC\#2 } & 2006 & Jun16-Jun18 & 981745281.62 & 1.73 & 0.16 & 2.10 & 119 & 11798 \\
\hline & 2007 & Jun02-Jun04 & 981745277.38 & 1.18 & 0.15 & 2.13 & 58 & 5718 \\
\hline & 2008 & Jun02-Jun04 & 981745272.62 & 1.22 & 0.12 & 2.15 & 96 & 9422 \\
\hline & 2012 & May31-Jun02 & 981745272.53 & 1.44 & 0.19 & 2.23 & 56 & 5473 \\
\hline & 2013 & Jun12-Jun15 & 981745259.39 & 2.53 & 0.25 & 2.13 & 104 & 12228 \\
\hline & 2015 & Jun14-Jun17 & 981745268.77 & 1.93 & 0.18 & 2.13 & 112 & 11051 \\
\hline \multirow[t]{6}{*}{ GBCL } & 2006 & Jun03-Jun05 & 981768410.16 & 1.48 & 0.15 & 2.08 & 92 & 9135 \\
\hline & 2007 & Jun04-Jun08 & 981768409.84 & 1.69 & 0.13 & 2.14 & 160 & 15952 \\
\hline & 2008 & Jun11-Jun13 & 981768398.93 & 1.81 & 0.17 & 2.13 & 116 & 11533 \\
\hline & 2012 & Jun04-Jun06 & 981768391.04 & 2.16 & 0.22 & 2.29 & 100 & 9846 \\
\hline & 2013 & Jun03-Jun04 & 981768384.24 & 3.00 & 0.32 & 2.17 & 90 & 8932 \\
\hline & 2015 & Jun05-Jun07 & 981768371.42 & 2.04 & 0.18 & 2.19 & 126 & 12527 \\
\hline \multirow[t]{6}{*}{ RUSG \#3 } & 2006 & Jun07-Jun09 & 981796822.55 & 2.16 & 0.23 & 2.13 & 89 & 8708 \\
\hline & 2007 & Jun10-Jun12 & 981796822.41 & 1.35 & 0.14 & 2.17 & 92 & 9134 \\
\hline & 2008 & Jun07-Jun10 & 981796815.62 & 1.49 & 0.15 & 2.14 & 94 & 9334 \\
\hline & 2012 & Jun09-Jun10 & 981796832.52 & 25.43 & 2.86 & 3.81 & 79 & 7752 \\
\hline & 2013 & Jun07-Jun08 & 981796791.54 & 4.00 & 0.40 & 2.58 & 100 & 9638 \\
\hline & 2015 & Jun09-Jun11 & 981796783.91 & 2.25 & 0.23 & 2.12 & 100 & 9894 \\
\hline \multirow[t]{6}{*}{ HNSG } & 2006 & Jun11-Jun13 & 981832008.48 & 1.58 & 0.14 & 2.16 & 123 & 12205 \\
\hline & 2007 & Jun14-Jun17 & 981832003.60 & 1.70 & 0.15 & 2.14 & 128 & 12698 \\
\hline & 2008 & Jun15-Jun18 & 981831997.90 & 1.71 & 0.15 & 2.10 & 124 & 12288 \\
\hline & 2012 & Jun17-Jun18 & 981831990.15 & 1.64 & 0.17 & 2.15 & 92 & 9126 \\
\hline & 2013 & Jun16-Jun18 & 981831977.17 & 2.54 & 0.29 & 2.17 & 75 & 8863 \\
\hline & 2015 & Jun17-Jun19 & 981831973.66 & 1.60 & 0.16 & 2.12 & 101 & 9992 \\
\hline \multirow[t]{5}{*}{ BRMC\#4 } & 2007 & Jun18-Jun20 & 981660211.75 & 1.47 & 0.15 & 2.15 & 94 & 9308 \\
\hline & 2008 & Jun19-Jun21 & 981660206.72 & 1.30 & 0.16 & 2.08 & 64 & 6297 \\
\hline & 2012 & Jun19-Jun21 & 981660201.73 & 1.50 & 0.16 & 2.14 & 90 & 8771 \\
\hline & 2013 & Jun19-Jun20 & 981660196.67 & 4.19 & 0.42 & 2.14 & 99 & 9776 \\
\hline & 2015 & Jun20-Jun22 & 981660186.23 & 2.08 & 0.20 & 2.07 & 113 & 10958 \\
\hline
\end{tabular}

\#1 SD, SE, UC, $N_{s}$ and $N_{d}$ indicate the standard deviation, standard error, uncertainty, set and drop numbers, respectively.

$\mathrm{SE}$ was calculated as the ratio of $\mathrm{SD}$ to the root of $N_{s}$. UC was estimated in the g9 software, by considering errors associated with instrumental systems and gravity data corrections.

\#2 The AG values at MGVC in 2012-2015 include the gravity difference between the old and new gravity points.

\#3 The AG value at RUSG in 2012 has large errors due to the failure of the long-period spring in the FG5 gravimeter.

\#4 No AG measurement was conducted at BRMC in 2006. 
Table 3. : The viscosity structure defined for RPIM and LPIM.

\begin{tabular}{c|cc|c|c}
\hline Layer & \multicolumn{2}{|c|}{ Radius $[\mathrm{km}]$} & Thickness $[\mathrm{km}]$ & Vicosity $\left[10^{19} \mathrm{~Pa} \mathrm{~s}\right]$ \\
& Bottom & Top & & \\
\hline Lithosphere & $6251-6341$ & 6371 & $30-120(=d)$ & Infinity \\
Asthenosphere 1 & 6151 & $6251-6341$ & $100-190$ & $0.5-5.0(=\eta)$ \\
Asthenosphere 2 & 5971 & 6151 & 180 & 40.0 \\
Asthenosphere 3 & 5701 & 5971 & 270 & 40.0 \\
Asthenosphere 4 & 3480 & 5701 & 2221 & 200.0 \\
Liquid core & 0 & 3480 & 3480 & 0.0 \\
\hline
\end{tabular}

\section{Figure captions}

\section{Figure 1}

AG points in SE-AK. Red circles and squares indicate the gravity points at which AG values were repeatedly measured in 2006-2015 and 2007-2015, respectively. The cyan areas and contour lines indicate the glacier distribution of the UAF05 PDIM model (Larsen et al 2007) and the rate of ground uplift in mm/yr (Larsen et al 2005) at intervals of $2 \mathrm{~mm} / \mathrm{yr}$, respectively. The green triangle indicates Long Lake's weather station belonging to the National Water and Climate Center, and dashed lines indicate the borders of countries and states.

\section{Figure 2}

The correction of the ocean tide loading effect from the AG data was collected at RUSG in 2008. (a) The set gravity data before the ocean loading effect was corrected (black circles) and the gravity change due to the ocean loading effect expected by Inazu et al. (2009) (red line). (b) The set gravity data after the ocean loading effect was corrected using the Inazu model (black circles). (c) The set gravity data after the ocean loading effect was corrected using the Inazu model (black circles) and the diurnal/semidiurnal regression curve for the gravity data (red line). (d) The set gravity data after the ocean loading effect was corrected using both the Inazu model and the diurnal/semidiurnal regression (black circles). In all the panels, the gray bars and green lines indicate the standard error and weighted average values of the set gravity data, respectively, and the vertical axis refers to the average value in 
panel (d), which is defined by $g_{0}$.

\section{Figure 3}

Gravity change associated with hydrological effect. (a) An orange line indicates the snow water equivalent (SWE) value observed at Long Lake's weather station (green triangle in Fig. 1), and a cyan line indicates the SWE value at the EGAN's gravity point obtained from the GLDAS-2.1 data-set (Rodell et al 2004). (b) Monthly gravity change at EGAN, calculated from the GLDAS-2.1 model (Rodell et al 2004). Colored lines in the top, middle, and bottom of the panel indicate the calculated gravity changes associated with mass variations in soil moisture, snow and canopy, respectively. Pink and green lines indicate the gravity changes due to the elastic loading deformation and mass attraction $\left(g_{e}\right.$ and $\left.g_{a}\right)$, respectively, and cyan lines indicate the sum of the two effects $\left(g_{e}+g_{a}\right)$. (c) Cyan lines indicate the hydrological gravity changes at six AG points in SE-AK, calculated as the sum of the loading/attraction effects due to soil moisture, snow and canopy (see panel (b)). In each panel, the circles indicate the values at the time when the AG values were measured.

\section{Figure 4}

Coseismic gravity changes in SE-AK. Panels (a) and (b) show the coseismic gravity changes due to the Haida Gwaii and Craig Earthquakes around SE-AK, respectively, which is estimated from the slip distributions by Lay et al (2013) and the calculation software by Okubo (1992). The green squares and red circles indicate the seismic faults defined by Lay et al (2013) and six AG points in Southeast Alaska, respectively. The epicenters (yellow stars) and source mechanism in these panels were analyzed by the Global CMT Project (http://www.globalcmt.org/). The panels (c) and (d) show the coseismic gravity changes due to the Haida Gwaii and Craig Earthquakes in SE-AK, respectively. Contours of the gravity changes are drawn every $0.1 \mu \mathrm{Gal}$, and the red circles indicate the AG points.

\section{Figure 5}

AG values and their variation rates at six AG points in SE-AK from 2006 to 2015. Green/red circles indicate the AG values before/after the effects of hydrological and coseismic gravity changes were corrected. An arbitrary constant value was subtracted from the AG values at each AG point, so as to draw all of the AG data in this figure, although the AG value at RUSG in 2012 was not shown because of the large error associated with the instrumental failure. The vertical bar for each red circle indicates the error value of the corresponding AG data calculated as the root sum square of SD and UC (see Table 2). The red line for each AG point indicates the regression line to the AG values measured from 
2006 to 2015 (red circles) and its variation rate with its standard deviation is shown as a red value on the right side of the figure. For the gravity point of MGVC, the amplitude of the gravity step between 2008 and 2012 was also calculated associated with the relocation of the gravity point in Mendenhall Glacier Visitor Center. Gray-colored values on the right side of the figure indicate the gravity variation rates determined in the earlier studies (Sun et al 2010; Sato et al 2012).

\section{FIgure 6}

The behavior of the Love numbers obtained from the VM5a model for GPIM (Peltier et al 2015). (a)

The variation in $-\dot{\delta}_{n}(\tau)$ with degree $(n)$ and time $(\tau)$. (b) The variation in $\dot{h}_{n}(\tau)$ with degree $(n)$ and time $(\tau)$. (c) The variation in the ratio of $-\dot{\delta}_{n}(\tau)$ to $\dot{h}_{n}(\tau)$ with degree $(n)$ and time $(\tau)$. (d) The variation in $-\dot{\delta}(\varphi, \tau)$ with angular distance $(\varphi)$ and time $(\tau)$. (e) The variation in $\dot{h}(\varphi, \tau)$ with angular distance $(\varphi)$ and time $(\tau)$. (f) The variation in the ratio of $-\dot{\delta}(\varphi, \tau)$ to $\dot{h}(\varphi, \tau)$ with angular distance $(\varphi)$ and time $(\tau)$

\section{Figure 7}

The distribution of $\chi^{2}$ to the variations of $d$ and $\eta$. The red area in panel (a) corresponds to the area shown in panel (b). In the panel (b), the red cross indicates the position where the $\chi^{2}$ value reaches a minimum, and the green line indicates the error range of $(\eta, d)$.

\section{Figure 8}

The behavior of the Love numbers used to calculate the response to LPIM and RPIM. The Love number for $\tau=2$ kyr determined from the VM5a model (orange lines in Fig. 6) is shown as the gray-colored symbol in each panel, for comparison with those obtained from the subsurface structure model (Table 3 and Fig. 7). (a) The variation in $-\dot{\delta}_{n}(\tau)$ with degree $(n)$ and time $(\tau)$. (b) The variation in $\dot{h}_{n}(\tau)$ with degree $(n)$ and time $(\tau)$. (c) The variation in the ratio of $-\dot{\delta}_{n}(\tau)$ to $\dot{h}_{n}(\tau)$ with degree $(n)$ and time $(\tau)$. (d) The variation in $-\dot{\delta}(\varphi, \tau)$ with angular distance $(\varphi)$ and time $(\tau)$. (e) The variation in $\dot{h}(\varphi, \tau)$ with angular distance $(\varphi)$ and time $(\tau)$. (f) The variation in the ratio of $-\dot{\delta}(\varphi, \tau)$ to $\dot{h}(\varphi, \tau)$ with angular distance $(\varphi)$ and time $(\tau)$.

\section{Figure 9}

The gravity variation rates at six AG points. Purple, blue, cyan, and green bars indicate the calculated gravity variation rates associated with GPIM, RPIM, LPIM, and PDIM, respectively. Orange, pink, yellow, and brown bars indicate the four components of the PDIM-derived gravity change (green bars); i.e., the orange/pink bars indicate the loading/attraction effects calculated from the UAF07's PDIM 
model. The yellow/brown bars indicate the loading/attraction effects calculated from the UAF05's PDIM model. Red triangles and green circles indicate the gravity variation rates calculated from our GIA model and determined from our AG measurement, respectively. Blue triangles and black circles indicate the gravity variation rates calculated and observed by the earlier studies (Sun et al 2010; Sato et al 2012), respectively. Yellow diamonds indicate the gravity variation rates obtained by reversing the sign of the attraction effect for the rate calculated by Sato et al (2012).

\section{Figure 10}

Vertical ground deformation rates for 99 GNSS stations in SE-AK. Panels (a) and (d) show the histogram and spatial distribution of the observed vertical ground deformation rates, respectively.

Panels (b) and (e) show the histogram and spatial distribution of the vertical ground deformation rates calculated from our GIA model, respectively. Panels (c) and (f) show the histogram and spatial distribution, respectively, of the residual between the observed and calculated vertical ground deformation rates, respectively. In panels (a)-(c), the red line and two green lines indicate the average and 1- $\sigma$ error range, respectively.

\section{Figure 11}

The ratio of gravity variation to vertical ground deformation at six AG points. Green circles and red triangles indicate the ratio values obtained from the observation data and calculated results, respectively. Green and red lines indicate the average values of the observed and calculated ratios, respectively, and these average values are shown on the bottom left of each panel. (a) Raw ratio. (b) Total ratio. (c) Viscous ratio. (d) Elastic ratio.

\section{Figure 12}

The variation in the optimal values of the rheological parameters when the scale factor for PDIM is changed from 0.5 to 2.0. Red and blue lines indicate the optimal lithospheric thickness $(d)$ and the upper mantle viscosity $(\eta)$, respectively, and their error ranges are shown as the gray areas. The stars indicate the optimal values of $(d, \eta)$ when the scale factor is 1.0 (Fig. 7 ).

\section{Figure A1}

The location of the MGVC gravity point. (a) The satellite image of Mendenhall Glacier Visitor Center derived from Google Earth Pro. The red circle and green triangle indicate the MGVC gravity point and AB50 GNSS station, respectively. The white arrow indicates the direction of the photo (b). (b) Entrance of Mendenhall Glacier Visitor Center. MGVC is located about $25 \mathrm{~m}$ from the entrance. (c) 
The elevator lobby of the Mendenhall Glacier Visitor Center, where the AG point is located. The new AG point is located on the right side of the lobby when facing the elevators. The old AG point (Sun et al 2010) was located at the left side of the lobby. However, in June 2012, we found that the old point was not available because of the illuminating exhibition. The white arrow indicates the photo direction of Figure A5 in Sun et al (2010). (d) The FG5-111 absolute gravimeter on the new AG point. (e) Position of new AG point. It is located at the tip of the pen and approximately $109.5 \mathrm{~cm}$ from the corner of the heater. The heater should not be activated during the AG measurement because its vibration magnifies the scatter of the AG data. (f) Exact position of the new AG point. It is located at the center of a tile and approximately $63.5 \mathrm{~cm}$ from the corner of the pillar.

\section{Figure A2}

The location of the BRMC gravity point. (a) The satellite image of the vicinity of BRMC derived from Google Earth Pro. The dashed line indicates the 60th parallel north, which corresponds to the border between the Yukon Territory (TY) and British Columbia (BC), Canada. When you access to BRMC (red circle), drive a van along the Haines Highway northward from Haines, and turn left after crossing over the Blanchard River. (b) Satellite image of BRMC derived from Google Earth Pro. The white line and yellow arrow indicate the access route to BRMC and the direction of the photo (c), respectively. BRMC (red circle) is located in the garage. (c) The garage of Blanchard Road Maintenance Compound. The white line indicates the access route to BRMC by car. The shutters at the tip of the white arrow can be opened when you transport AG instruments closely to the gravity point. (d) The FG5-111 absolute gravimeter on the AG point. (e) The exact position of the AG point. It is located about 129.3 $\mathrm{cm}$ from the corner of the gutter, and about $220.5 \mathrm{~cm}$ from the corner of the red door. (f) Close-up photo of the AG point. This AG point was originally indicated with scratch marks of a small cross and a large square in and before 2012. However, the garage floor was painted by compound staff after the AG measurement in June 2012. Since we could not easily find the marks under the painting in June 2013, we highlighted the marks with a permanent marker for future AG measurements. 


\section{Figures}

\section{Figure 1}

AG points in SE-AK. Red circles and squares indicate the gravity points at which AG values were repeatedly measured in 2006-2015 and 2007-2015, respectively. The cyan areas and contour lines indicate the glacier distribution of the UAF05 PDIM model (Larsen et al 2007) and the rate of ground uplift in mm/yr (Larsen et al 2005) at intervals of $2 \mathrm{~mm} / \mathrm{yr}$, respectively. The green triangle indicates Long Lake's weather station belonging to the National Water and Climate Center, and dashed lines indicate the borders of countries and states.

\section{Figure 2}

The correction of the ocean tide loading effect from the AG data was collected at RUSG in 2008. (a) The set gravity data before the ocean loading effect was corrected (black circles) and the gravity change due to the ocean loading effect expected by Inazu et al. (2009) (red line). (b) The set gravity data after

751 the ocean loading effect was corrected using the Inazu model (black circles). (c) The set gravity data after the ocean loading effect was corrected using the Inazu model (black circles) and the diurnal/semidiurnal regression curve for the gravity data (red line). (d) The set gravity data after the ocean loading effect was corrected using both the Inazu model and the diurnal/semidiurnal regression(black circles). In all the panels, the gray bars and green lines indicate the standard error and weighted average values of the set gravity data, respectively, and the vertical axis refers to the average value in panel (d), which is defined by $g_{0}$.

\section{Figure 3}

Gravity change associated with hydrological effect. (a) An orange line indicates the snow water equivalent (SWE) value observed at Long Lake's weather station (green triangle in Fig. 1), and a cyan line indicates the SWE value at the EGAN's gravity point obtained from the GLDAS-2.1 data-set (Rodell et al 2004). (b) Monthly gravity change at EGAN, calculated from the GLDAS-2.1 model (Rodell et al 2004). Colored lines in the top, middle, and bottom of the panel indicate the calculated gravity changes associated with mass variations in soil moisture, snow and canopy, respectively. Pink and green lines indicate the gravity changes due to the elastic loading deformation and mass attraction (ge and ga), respectively, and cyan lines indicate the sum of the two effects (ge + ga). (c) Cyan lines indicate the hydrological gravity changes at six AG points in SE-AK, calculated as the sum of the loading/attraction 
effects due to soil moisture, snow and canopy (see panel (b)). In each panel, the circles indicate the values at the time when the $A G$ values were measured.

\section{Figure 4}

Coseismic gravity changes in SE-AK. Panels (a) and (b) show the coseismic gravity changes due to the Haida Gwaii and Craig Earthquakes around SE-AK, respectively, which is estimated from the slip distributions by Lay et al (2013) and the calculation software by Okubo (1992). The green squares and red circles indicate the seismic faults defined by Lay et al (2013) and six AG points in Southeast Alaska, respectively. The epicenters (yellow stars) and source mechanism in these panels were analyzed by the Global CMT Project (http://www.globalcmt.org/). The panels (c) and (d) show the coseismic gravity changes due to the Haida Gwaii and Craig Earthquakes in SE-AK, respectively. Contours of the gravity changes are drawn every $0.1 \mu \mathrm{Gal}$, and the red circles indicate the AG points.

\section{Figure 5}

AG values and their variation rates at six AG points in SE-AK from 2006 to 2015 . Green/red circles indicate the AG values before/after the effects of hydrological and coseismic gravity changes were corrected. An arbitrary constant value was subtracted from the AG values at each AG point, so as to draw all of the AG data in this figure, although the AG value at RUSG in 2012 was not shown because of the large error associated with the instrumental failure. The vertical bar for each red circle indicates the error value of the corresponding AG data calculated as the root sum square of SD and UC (see Table 2). The red line for each AG point indicates the regression line to the AG values measured from 2006 to 2015 (red circles) and its variation rate with its standard deviation is shown as a red value on the right side of the figure. For the gravity point of MGVC, the amplitude of the gravity step between 2008 and 2012 was also calculated associated with the relocation of the gravity point in Mendenhall Glacier Visitor Center. Graycolored values on the right side of the figure indicate the gravity variation rates determined in the earlier studies (Sun et al 2010; Sato et al 2012).

\section{Figure 6}

See image above for figure legend

\section{Figure 7}


Figure 8

See image above for figure legend

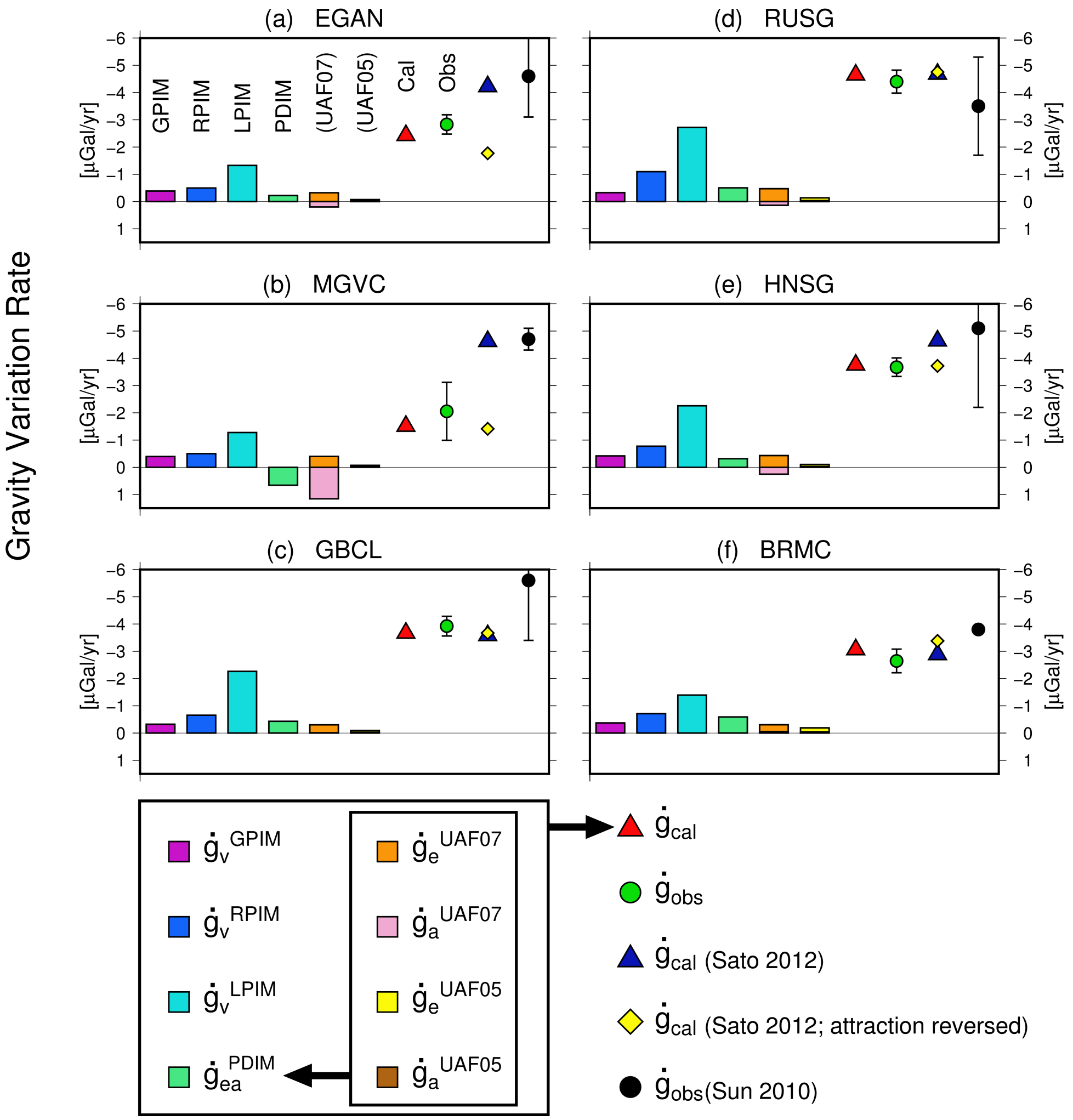




\section{Figure 9}

The gravity variation rates at six AG points. Purple, blue, cyan, and green bars indicate the calculated gravity variation rates associated with GPIM, RPIM, LPIM, and PDIM, respectively. Orange, pink, yellow, and brown bars indicate the four components of the PDIM-derived gravity change (green bars); i.e., the orange/pink bars indicate the loading/attraction effects calculated from the UAF07's PDIM model. The yellow/brown bars indicate the loading/attraction effects calculated from the UAF05's PDIM model. Red triangles and green circles indicate the gravity variation rates calculated from our GIA model and determined from our AG measurement, respectively. Blue triangles and black circles indicate the gravity variation rates calculated and observed by the earlier studies (Sun et al 2010; Sato et al 2012), respectively. Yellow diamonds indicate the gravity variation rates obtained by reversing the sign of the attraction effect for the rate calculated by Sato et al (2012).

\section{Figure 10}

Vertical ground deformation rates for 99 GNSS stations in SE-AK. Panels (a) and (d) show the histogram and spatial distribution of the observed vertical ground deformation rates, respectively. Panels (b) and (e) show the histogram and spatial distribution of the vertical ground deformation ratescalculated from our GIA model, respectively. Panels (c) and (f) show the histogram and spatial distribution, respectively, of the residual between the observed and calculated vertical ground deformation rates, respectively. In panels (a) $-(\mathrm{c})$, the red line and two green lines indicate the average and 1- $\sigma$ error range, respectively.

\section{Figure 11}

The ratio of gravity variation to vertical ground deformation at six AG points. Green circles and red triangles indicate the ratio values obtained from the observation data and calculated results, respectively. Green and red lines indicate the average values of the observed and calculated ratios, respectively, and these average values are shown on the bottom left of each panel. (a) Raw ratio. (b) Total ratio. (c) Viscous ratio. (d) Elastic ratio. 


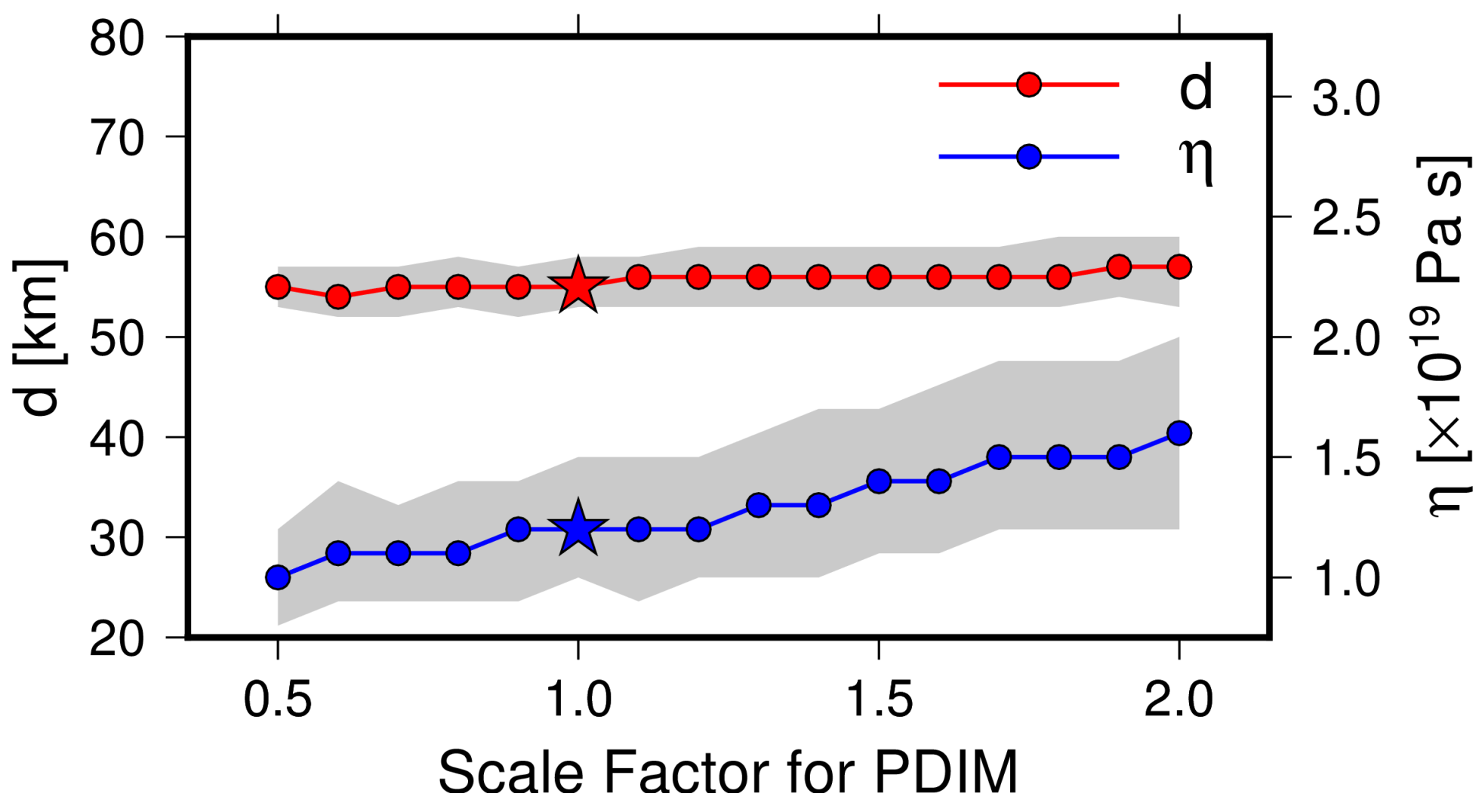

Figure 12

The variation in the optimal values of the rheological parameters when the scale factor for PDIM is changed from 0.5 to 2.0. Red and blue lines indicate the optimal lithospheric thickness (d) and the upper mantle viscosity $(\eta)$, respectively, and their error ranges are shown as the gray areas. The stars indicate the optimal values of $(d, \eta)$ when the scale factor is 1.0 (Fig. 7).

\section{Supplementary Files}

This is a list of supplementary files associated with this preprint. Click to download.

- FigA1MGVC.png

- Abstract.png

- FigA2BRMC.png 